\title{
Anticipation via canards in excitable systems
}

\author{
Elif Köksal Ersöz, ${ }^{1}$, a) Mathieu Desroches, ${ }^{1}$ Claudio R. Mirasso, ${ }^{2}$ and Serafim Rodrigues ${ }^{3,4, b)}$ \\ ${ }^{1)}$ MathNeuro team, Inria Sophia Antipolis Méditerranée, 06902 Sophia Antipolis, \\ France \\ ${ }^{2)}$ Instituto de Física Interdisciplinar y Sistemas Complejos IFISC (CSIC-UIB), \\ Universitat de les Illes Baleares, Campus UIB, E-07122 Palma de Mallorca, \\ Spain \\ 3) IKERBASQUE: The Basque Foundation for Science, 48013 Bilbao, Basque Country, \\ Spain \\ ${ }^{4)} B C A M$ - Basque Centre for Applied Mathematics, Mazarredo, 14 E48009 Bilbao, \\ Basque Country - Spain.
}

Neurons can anticipate incoming signals by exploiting a physiological mechanism not well understood. This article offers a novel explanation on how a receiver neuron can predict the sender's dynamics in a unidirectionallycoupled configuration, in which both sender-receiver follow the evolution of a multi-scale excitable system. We present a novel theoretical view point based on a mathematical object, called canard, to explain anticipation in excitable systems. We provide a numerical approach, which allows to determine the transient effects of canards. To demonstrate the general validity of canard-mediated anticipation in the context of excitable systems, we illustrate our framework in two examples, a multi-scale radio-wave circuit (the van der Pol model) that inspired a caricature neuronal model (the FitzHugh-Nagumo model) and a biophysical neuronal model (a 2-dimentional reduction of the Hodgkin-Huxley model), where canards act as messengers to the senders' prediction. We also propose an experimental paradigm that would enable experimental neuroscientists to validate our predictions. We conclude with an outlook to possible fascinating research avenues to further unfold the mechanisms underpinning anticipation. We envisage that our approach can be employed to a wider class of excitable systems with appropriate theoretical extensions.

Anticipation appears as a counter-intuitive observation in a wide range of dynamical systems ranging from biology to engineering applications. It can occur in unidirectionally coupled systems when the receiver is subject to a self-delayed feedback in addition to a signal coming from the sender. This particular interaction permits the receiver to predict the future trajectory of the sender. Anticipation can occur transiently, thus straightforwardly denoted anticipation, or in long-term dynamics, in which case it is referred to as anticipated synchronization. In this study, we focus on both aspects of anticipatory dynamics in the context of excitable systems and explain it via a counter-intuitive phenomenon, namely canards. Canard trajectories structure the excitability and synchronization properties of multiple timescale systems exhibiting excitable dynamics. By developing a theoretical framework enhanced by numerical continuation, we show that the underlying canard structure in excitable systems is responsible for delaying sub-threshold solutions, but anticipating the spiking ones. We also propose an experimental set up that would enable experimentalists to observe anticipated behavior in neural systems, in particular in type-II neurons.

\footnotetext{
a) Electronic mail: elif.koksal@inria.fr.

b) Electronic mail: srodrigues@bcamath.org.
}

\section{INTRODUCTION AND MOTIVATION}

Anticipated synchronization (AS), or more generally anticipation, is a counter-intuitive dynamical behavior that can occur between two unidirectionally coupled systems. Unexpectedly, under certain conditions, the state of a receiver system anticipates that of the sender. This phenomenon was first discovered by Voss in unidirectionally coupled chaotic oscillators ${ }^{1}$. Voss proposed two altenative schemes ruled by the following equations:

$$
\begin{aligned}
& \dot{\mathbf{x}}=-\alpha \mathbf{x}(t)+\mathbf{F}(\mathbf{x}(t-\tau)) \\
& \dot{\mathbf{y}}=-\alpha \mathbf{y}(t)+\mathbf{F}(\mathbf{x}(t))
\end{aligned}
$$

or

$$
\begin{aligned}
\dot{\mathbf{x}} & =\mathbf{F}(\mathbf{x}(t)) \\
\dot{\mathbf{y}} & =\mathbf{F}(\mathbf{y}(t))+\mathbf{K}(\mathbf{x}(t)-\mathbf{y}(t-\tau))
\end{aligned}
$$

where the vector $\mathbf{x}$ corresponds to the variables of the master system, $\mathbf{y}$ corresponds to those of the slave system, $\mathbf{F}$ is a vector function. The first scheme is called complete replacement and the second one delay coupling. In (2) $\mathbf{K}$ is the coupling matrix between the sender $\mathbf{x}$ and the receiver $\mathbf{y}$, and $\tau$ is the delay time. Note that $\mathbf{y}(t)=\mathbf{x}(t+\tau)$ is a solution of both (1) and (2). Moreover, Voss showed that these solutions can be stable for certain parameter values. For the case of complete replacement, the anticipation time can be arbitrarily large; for the delay coupling, some constraints on the anticipation time and coupling $\mathbf{K}$ are required for the synchronization solution to be stable. If an external forcing $I(t)$, which is neither constant nor periodic with period $\tau$, is added to 
the set of equations $(2), \mathbf{y}(t)=\mathbf{x}(t+\tau)$ is no longer an exact solution of the problem. However, it has been numerically shown that the system tends to operate in the AS regime (see, e.g. Refs. 2 and 3). Furthermore, it can occur transiently in the excitable regime ${ }^{4}$.

The theoretical framework as proposed by Voss, in particular for the delay coupling scheme, has led to an intense subject of research (theoretically and experimentally) over the past 15 years due to an apparent violation of causality, thus contradicting a central postulate that underpins Science and Engineering. In particular within Neuroscience, a number of studies have shown that anticipation occurs in electrically coupled excitable neuron models ${ }^{2,4}$, and in synaptically coupled neuron models $^{3,5-7}$. The anticipation phenomena appear to be robust and a particular example was shown by Pyragienè and Pyragas ${ }^{8}$ for chaotic neuronal models, where the receiver anticipates the chaotic neuronal spikes (i.e. electrical activations of neurons) of the sender neuron. Voss' scheme of (2) was used to explain the estimations of a positive Granger Causality, with well-defined directional influence, accompanied by either a positive or negative phase lag in the recordings of the motor cortex activity of monkeys while performing a visual task ${ }^{6}$. AS has also been applied to control and parameter estimation problems in dynamical systems both numerically ${ }^{9-12}$ and experimentally ${ }^{6,13-18}$.

A number of theoretical and numerical methods have been developed to unveil the mechanism of anticipation. Starting with the work by Voss in systems of the form (1) and (2). A particular implementation of the replacement scheme, denoted subsystem decomposition, which considers the decomposition dynamics of the sender system was originally developed by Refs. 19 and 20 and later explored by various researchers. Other methods include phase-lag compensating coupling ${ }^{21}$, phase response curves (PRC) and improvements by considering maps based on $\mathrm{PRC}^{22}$. Although the understanding of anticipation has advanced considerably, a complete knowledge of the underlying mechanism is still lacking. To shed light into the basic mechanisms, the present paper focuses on multi-timescale systems exhibiting excitable dynamics, which is ubiquitous in complex systems (e.g. neurons) and further extend the understanding of AS by providing a novel theoretical explanation.

The starting point of our theoretical study derives through the culmination of three key developments and observations: (1) The theoretical work by Ciszak et al. ${ }^{4}$ linked anticipation in excitable systems to a reduction of excitability threshold. (2) Previous studies of some of the authors of the present manuscript have demonstrated that the excitability threshold is associated to a special kind of trajectories that are sensitive to perturbations, the so-called canard solutions ${ }^{23}$ (details will be clarified in subsequent paragraphs). This suggests canards as the key mechanism mediating anticipation in excitable systems, and provides a theoretical extension of the results by Ciszak et al. ${ }^{4}$. Our recent work extended the infinitesimal Phase Response Curve (iPRCs), a method that was originally developed for analyzing small perturbation of a limit cycle trajectory, to the canard regime ${ }^{24}$. Based on these results, we demonstrate that in excitable systems, the mystery lies in the delayed coupling between the sender and receiver, which endows the receiver with an extra timescale separation therefore triggering faster canard trajectories that predict the senders trajectories. This enables the receiver to 'pre-synchronize' to the sender, thus, giving an illusion of anticipation. Our result shows that there is no violation of causality, thus rescuing the well defined (and expected) Scientific and Engineering views. Despite the fact that there is no violation of causality, it is fascinating to gauge that nature (i.e. neurons) exploit this mechanism for possibly efficiency reasons and as a consequence, paradoxically performing at speeds higher than expected. In addition, we believe that the unfolding of this mechanism will enable computational models to exploit anticipation across brain scales, thus establishing the link between neuronal node activations and that of large-scale neuronal circuits (associated to cognition).

To outline our results we conveniently organize the paper as follows: In the Methods section, we revisit the canard phenomenon and overview its importance in complex systems. We highlight the main mathematical framework employed in this paper, which includes a brief discussion on the period function along the canard explosion, its relation to the adjoint solution (or equivalently, the iPRCs) and discuss mathematical reduction techniques of an one-dimensional delayed signal. In the Results section, we show how to combine the aforementioned frameworks with our previous results in order to explain, by means of arc-length numerical continuation ${ }^{25}$, the causal link between canard and anticipation. We showcase the results in two systems. On the one hand, we consider the van der Pol (VDP) system (a radio-wave circuit) for a pedagogical reason because it is a canonical (parsimonious) model that allows the reader to easily grasp the concept of canard-mediated anticipation, and also because it is a variant of the FitzHugh-Nagumo phenomenological neuron model. The second example is a minimal biophysical neuronal model, namely a 2dimensional (2D) reduction of the Hodgkin-Huxley $(\mathrm{HH})$ system. This leads us to propose a potential experimental setup that would enable the observation of anticipation in neuronal circuits and would also confirm our theoretical predictions. To close up, we discuss our results on anticipation in light of supposed violation of causality. And finally, we summarize our findings and draw future perspectives in the Conclusion section. 


\section{METHODS}

\section{A. Canard phenomena in multi-timescale excitable systems}

Multiple timescale dynamical systems (alternatively, denoted slow-fast systems) are ubiquitous in science and engineering since they have the power to synthetically explain empirical data exhibiting complex dynamics. These complex dynamics occur due to strong interaction of system variables across various timescales that can be mathematically explained by the so-called canard solutions. This fundamental mathematical object was first discovered by a group of french mathematicians while analysing a radio-wave circuit, the VDP system, under a constant forcing ${ }^{26}$. The key finding was that multi-timescale systems of this type possess an underlying locally-invariant repulsive manifold, which unexpectedly causes solution trajectories (e.g. oscillations in the case of VDP) to dwell along the manifold for a time period without being repelled. To best expose these canard trajectories and show that they also underpin anticipated synchronization we guide the reader through them in the VDP model, whose equations are as follows:

$$
\begin{aligned}
\varepsilon \dot{x} & =y-\frac{x^{3}}{3}+x=: f(x, y) \\
\dot{y} & =c-x=: g(x, y)
\end{aligned}
$$

where $x$ is the fast variable, $y$ is the slow variable, $0<$ $\varepsilon \ll 1$ is the timescale parameter and $c$ is the bifurcation parameter. For $\varepsilon=0$, system (3) has a S-shaped fast nullcline or critical manifold $S_{0}=\{f(x, y)=0\}=\{y=$ $\left.x^{3} / 3-x\right\}$, with two fold points $\left(x_{f}^{-}, y_{f}^{-}\right)$and $\left(x_{f}^{+}, y_{f}^{+}\right)$ where $f_{x}\left(x_{f}^{ \pm}, y_{f}^{ \pm}\right)=(\partial f / \partial x)\left(x_{f}^{ \pm}, y_{f}^{ \pm}\right)=0$. At these fold points, the critical manifold changes its behavior, i.e., it is attracting for $x \in\left(-\infty, x_{f}^{-}\right) \cap\left(x_{f}^{+}, \infty\right)$ and repelling for $x \in\left(x_{f}^{-}, x_{f}^{+}\right)$.

System (3) exhibits a Hopf bifurcation when the slow nullcline $\{x=c\}$ crosses any of the fold points, that is at $c=c_{H}= \pm 1$, which creates limit cycle solutions. At an $O(\varepsilon)$-distance from $c_{H}$, the family of limit cycles undergoes a canard explosion ${ }^{27}$, that is, there exists a parameter value $c_{c}$ called canard value and the corresponding cycles grow to an $O(1)$ amplitude within a variation of this value that is exponentially small with respect to the timescale separation parameter $\left(O\left(\mathrm{e}^{-k / \varepsilon}\right)\right.$, with $k$ a positive real constant). Cycles along this explosive part of the family are termed canards $\left(\gamma_{\varepsilon, c}\right)$. A key feature of these canard trajectories is that they pass from the vicinity of one of the attracting branches of $S_{0}$ flowing along a so-called attracting slow manifold, to the vicinity of its repelling branch flowing along a repelling slow manifold. In this process, they come close to one of the fold points $\left(x_{f}^{ \pm}, y_{f}^{ \pm}\right)$. The counterintuitive repelling slow movement of canard cycles can be seen as an inertia of the system. To give an intuitive view, one can imagine a point moving on a canard trajectory as a football (see Fig. 1) rolling along the upper crest of a curved field for a dwelling time period before ending up in the lower crest of the field. This energy-like viewpoint is presented in Fig. 1 where every panel is associated with a specific cycle of VDP's periodic regime: (a) canard without head, (b) maximal canard, (c) canard with head and (d) relaxation oscillation. Each panel contains three plots: at the bottom, the time series of the corresponding cycle of interest; in the middle, a phase-plane representation $^{28}$ of the cycle together with the critical manifold $S_{0}$; at the top, the energy-like surface $S_{E}$ in $(x, y, E)$ space, defined by the equation $E=-\int f(x, y) d x$, onto which the critical manifold is projected together with the corresponding cycle. This particular representation illustrates very well the specificity of canards as cycles, stable along the canard explosion of VDP, which "surf" for a long time on top of an unstable crest of the energy surface. More specifically, in the VDP system, and generically in $2 \mathrm{D}$ slow fast systems with a similar geometry of nullclines, the canard phenomenon explains the transition from Hopf cycles with $O(\varepsilon)$-amplitude to relaxation cycles $\gamma_{\varepsilon, c}^{\mathrm{ro}}$ with $O(1)$-amplitude in a very brutal yet continuous process. Within an exponentially-small parameter variation this continuous transition involves two canard families, denoted respectively canards without head $\left(\gamma_{\varepsilon, c}^{\mathrm{nh}}\right)$ and canards with head $\left(\gamma_{\varepsilon, c}^{\mathrm{h}}\right)$, the boundary between these two families corresponding to the maximal canard $\left(\gamma_{\varepsilon, c}^{\mathrm{m}}\right)$. Canards without head subsequently follow an attracting slow manifold and then a repelling slow manifold until they escape from it towards the same attracting slow manifold that they initially followed. In contrast, canards with head terminate their repelling segment by escaping towards another attracting slow manifold, near the opposite branch of $S_{0}$. The boundary behavior is that of the maximal canard, that is, the canard without head with the longest repelling segment: it stays close to the repelling branch of $S_{0}$ in between its two fold points $\left(x_{f}^{ \pm}, y_{f}^{ \pm}\right)$. The maximal canard exists at a unique parameter value for which an expansion in $\varepsilon$ can be computed, namely $c_{c}=c_{H}-(1 / 8) \varepsilon-(3 / 32) \varepsilon^{2}+O\left(\varepsilon^{3}\right)$. Since it possesses the longest repelling segment, it also has the longest period among the canard cycles. Note that, in type-II neuron models with multiple timescales, the repelling segment of the maximal canard offers the best approximation to the excitability threshold. Past the canard with head regime, relaxation cycles $\left(\gamma_{\varepsilon, c}^{\mathrm{ro}}\right)$ are observed. These are cycles that follow attracting slow manifolds near the outer branches of $S_{0}$ without following any repelling slow manifold, that is, they jump when passing near each fold point of $S_{0}$.

As explained above, along the canard explosion the amplitude (or $\mathrm{L}_{2}$-norm) of the cycles increases sharply; this sharp increase is considered as a signature of the canard explosion in VDP-type systems. Yet another characteristic of the canard explosion is the behavior of the period function (see below) associated with this branch of cycles, which for the readers convenience we explain in the subsequent section since we employ it in this work. 
(a)

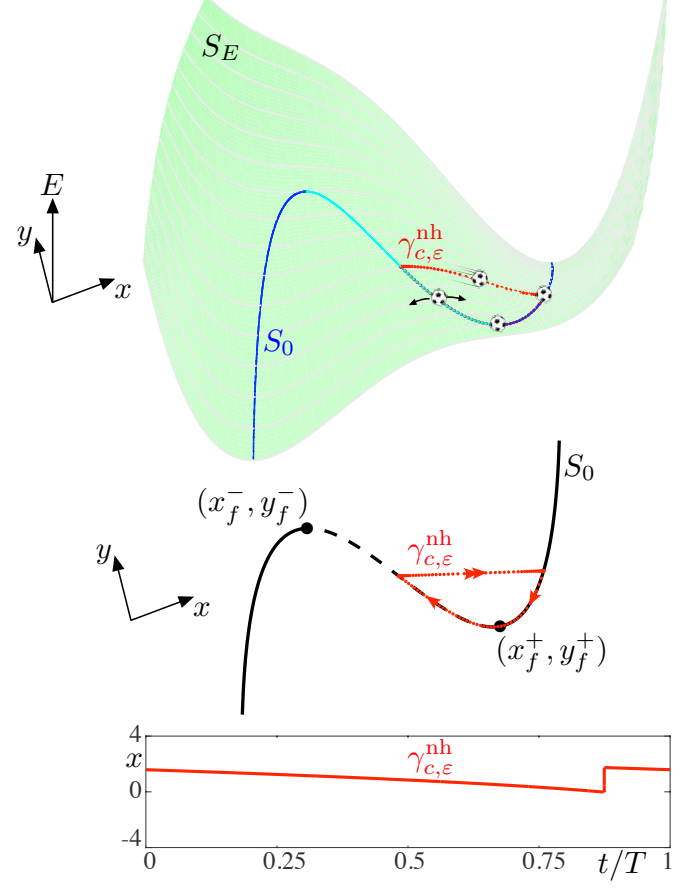

(c)

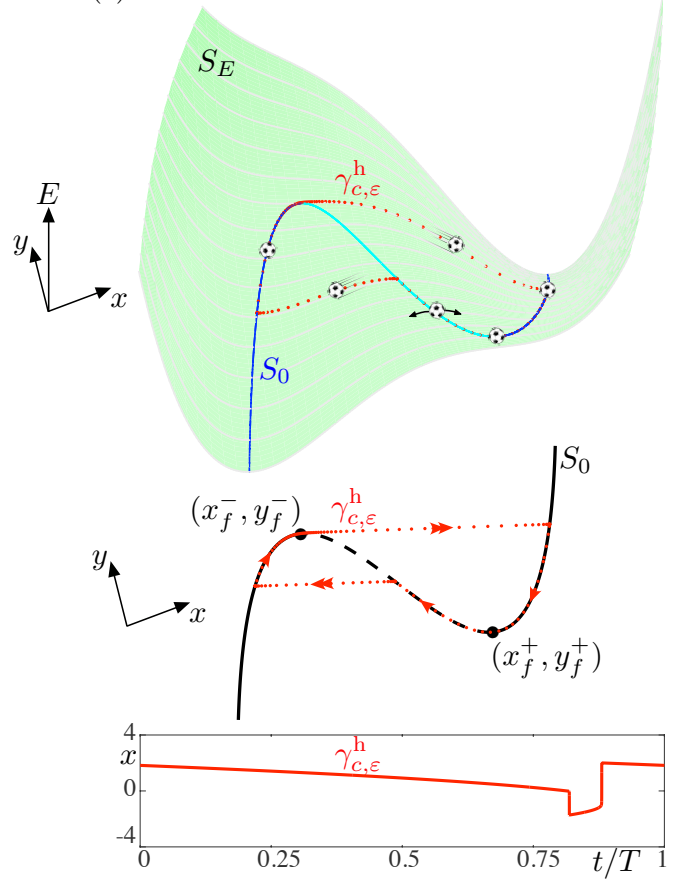

(b)
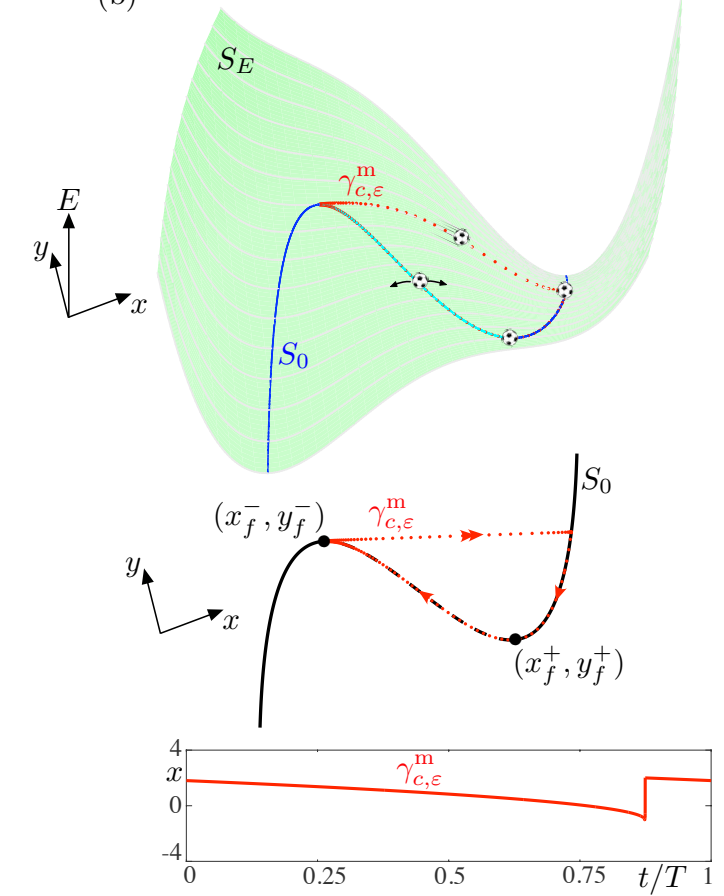

(d)

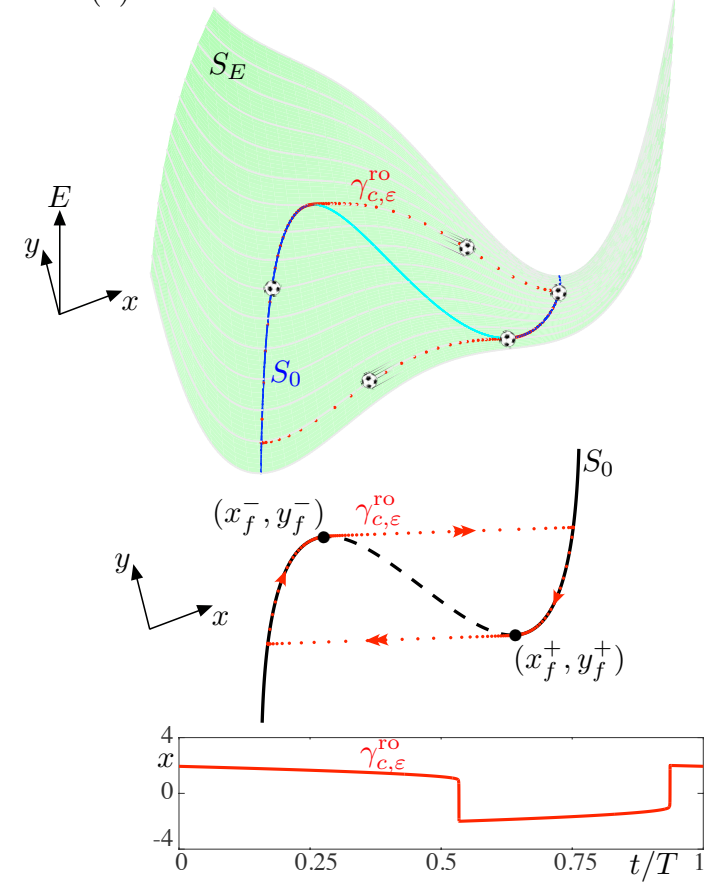

FIG. 1. Canard explosion from the energy viewpoint: Canard explosion ((a)-(c)) leading to relaxation oscillations ((d)) in the VDP system. On top of time series (lower part) and phase-plane projections (middle part), we show in the top part of each panel the energy-like surface $S_{E}$ (green), the critical manifold $S_{0}$ (blue-cyan on $S_{E}$, black on the phase-plane projections) as well as a limit cycle $\gamma_{c, \varepsilon}^{h}$ (red). Blue and solid black parts of $S_{0}$ are the attracting, cyan and dashed black parts of $S_{0}$ are the repelling branches of $S_{0}$. One-headed arrows indicate the slow motion, and two-headed arrows indicate the fast motion along the limit cycle.

\section{B. Period function of the canard explosion}

rameter value $c$ (for the $\operatorname{VDP}$ system, $c \in(-1,1))$, the

The period function $T_{\varepsilon}: C_{p}\left([0,1] ; \mathbb{R}^{n}\right) \times(-1,1) \rightarrow \mathbb{R}^{\geq 0}$ associates to any limit cycle $\gamma_{\varepsilon, c}$ corresponding to a pa- 
period $T_{\varepsilon}\left(\gamma_{\varepsilon, c}\right)$; here $C_{p}\left([0,1] ; \mathbb{R}^{n}\right)$ denotes the space of periodic functions defined on the unit interval. Importantly, it is known that in the canard regime, the period function is non-monotonic since, as explained above, the maximal canard has maximal period. This is exemplified in Fig 2, which depicts a branch of canard cycles in the VDP system for $\varepsilon=0.1$ as $c$ decreases from the Hopf bifurcation point at $c_{H}=1$. Between $c_{H}$ and the maximal canard at $c_{\text {max }} \approx 0.9863158$, the period of the headless canard cycles increases as $c$ decreases; see the bottom inset in panel (a) and the corresponding cycle in panel (b1). For $c<c_{\max }$ the system enters into the canardwith-head regime where the period decreases as the cycles grow; see the top inset in panel (a) and corresponding cycles in panel (a1). The non-monotonic behavior of the period function can be considered as a key aspect of the canard explosion. The maximum of the period function can be used to detect numerically the maximal canard trajectory, as a limit point on the branch obtained when the period is the main continuation parameter ${ }^{29}$.

\section{Infinitesimal PRCs and relation to the period function}

The response of canard cycles to perturbations can be exploited by using the non-monotonic behavior of the period function $T_{\varepsilon}$ along the canard explosion. A complementary key tool is the iPRC, which is a periodic function that quantifies the response in phase variations of a limit cycle solution $\gamma$ in the limit of infinitesimal perturbations ${ }^{24,30}$. The iPRC is determined by estimating the response variations $(\Delta \theta)$ of the phase $\theta: \gamma \rightarrow$ $[0, T]$ defined along the cycle, with $d \theta(\gamma(t)) / d t=1 / T$ where $T$ is the period of $\gamma$ and $\theta$ at $t=0$ is chosen arbitrarily, under $\varepsilon$-perturbations in the unit vector direction $\eta$. That is

$$
\lim _{\varepsilon \rightarrow 0} \frac{\Delta \theta}{\varepsilon}=D \theta(\gamma) \cdot \eta
$$

where the gradient of the phase function, $Z(t):=D \theta(\gamma)$, defines the iPRC. Additionally, the iPRC is the solution of the following adjoint variational equation

$$
\frac{Z(t)}{d t}+A(t)^{T} Z(t)=0,
$$

where $A(t)=D F(\gamma(t))$ is the linearisation of the vector field $F=(f, g)$ under consideration along the limit cycle $\gamma$; see details in Refs. 24 and 30. In particular, we are interested in using the iPRC with perturbation in the form of inputs from neighboring oscillators (in our case a single sender system) under a weak-coupling assumption.

In Fig 2 we exemplify the use of the iPRC and the period function. We underline two key points: (1) Slow manifolds, some defining the excitability threshold, and the maximal canard determine the response to infinitesimal perturbations. (2) The non-monotonic behavior of the period function along the canard explosion determines the response of a canard cycle to the perturbation received in the fold neighborhood. Panels (c1, c2) of Fig 2 illustrate that the $x$-component of the adjoint solutions gives access to the iPRC of the red canard cycles in (b1, b2), respectively. When an infinitesimal perturbation in the positive $x$-direction to the right of the fold is received by a canard-with-head cycle (b1), it is driven towards a larger cycle (like the yellow one in (b1)) with a shorter period. Consequently, the perturbed trajectory's phase is advanced as confirmed by the positive part of $Z_{1}(t)$ in panel $(\mathrm{c} 1) . Z_{1}(t)$ becomes negative when the trajectory $(x(t), y(t))$ crosses the lower fold $(x=1)$ while the trajectory moves away from the fold region. Thus, a small perturbation on the trajectory in this repelling region delays the phase transiently to a smaller but slower cycle, as exemplified by the blue curve in (b1). The delaying effect of the slow manifolds associated with canard trajectories extends the discussion by Ciszak et al. ${ }^{4}$ where the anticipation in excitable systems is interpreted as a lowering of the excitability threshold.

Similar phase-plane analysis applies to a small red canard cycle (b2). When perturbed in the attracting slow manifold near the fold region, it is driven transiently to a cycle with longer period, like the yellow one in (b2). Consequently the perturbed trajectory's phase is delayed compared to the unperturbed one, which corresponds to the negative part of the adjoint solution $Z_{1}(t)$ in Panel (c2). The sign on $Z_{1}(t)$ changes at the fold $x=1$ and becomes positive as the trajectory $(x(t), y(t))$ continues along the repelling branch. The trajectory's phase is advanced when the perturbation is received on the repelling slow manifold as indicated by this positive segment of $Z_{1}(t)$. In such a case, a perturbed trajectory transiently follows a smaller cycle, like the blue one in (b2), with a shorter period. In later sections we will show that a strong enough perturbation received either by a small canard cycle or when the system is at an equilibrium (possibly in excitable regime) can lead to a relaxation oscillation. This extreme regime is beyond the validity of the weak-coupling theory in the canard regime.

\section{Relevance of canards in general}

Within the field of mathematics, canards are recognised as a fundamental mathematical object and theoretical advances are being made to fully understand complex patterns mediated by canards in high-dimensional multiscale systems. Some of these theoretical techniques include, e.g. nonstandard analysis ${ }^{26,31}$, matched asymptotic expansions ${ }^{32}$ and blow-up methods ${ }^{33,34}$.

While being interesting mathematical objects, canards have direct applicability in engineering and applied sciences, and most interestingly in Neuroscience. For example, in lab experiments involving neurons, experimental data observations (via acquisition devices) show complex patterns and counter-intuitive rapid transitions via modulation of a control input, e.g. through electrical current injection. Furthermore, these observations show sensi- 

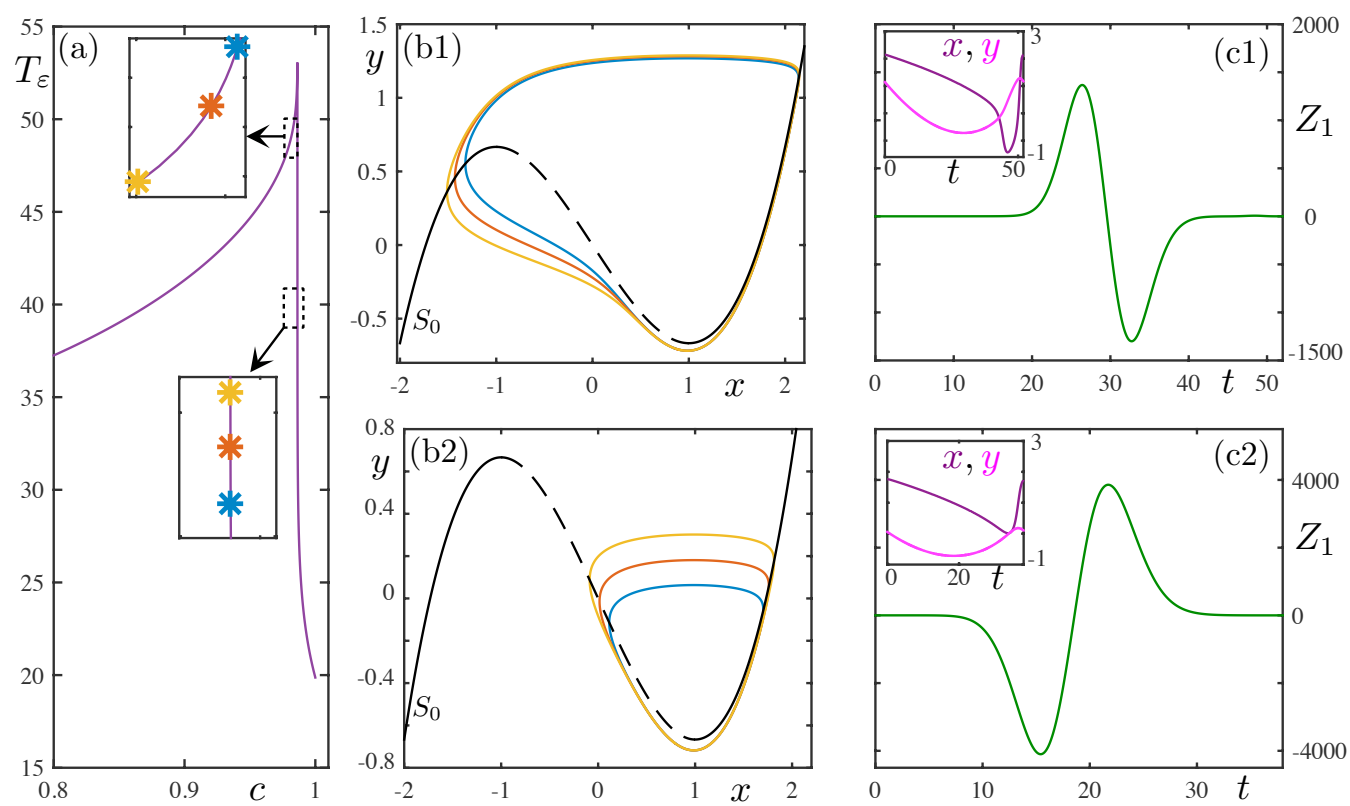

FIG. 2. Canard cycles and adjoint solutions: (a) Period of limit cycles along the canard explosion in the VDP system for $\varepsilon=0.1$; the parameter that varies is $c$. The period is increasing along the headless canard part of the branch, it reaches its maximum at the maximal canard and then decreases along the canard-with-head cycles. Top inset in (a): Periods of the three canard-with-head cycles shown in panel (b1) and marked on the period curve. Bottom inset in (a): Periods of three canardwithout-head cycles shown in panel (b2) and marked on the period curve. (b1) Three canard-with-head cycles corresponding to the top inset in panel (a). Larger cycles have shorter periods. (b2) Three canard-without-head cycles corresponding to the bottom inset in panel (a). Larger cycles have longer periods. Also shown in panels (b1) and (b2) is the critical manifold $S_{0}$, on which solid (resp. dashed) parts represent stable (resp. unstable) branches. (c1) First component of the adjoint solutions and $(x(t), y(t))$ ((inset), purple and pink lines, respectively) during one cycle of the red canard solutions in panel (b1). (c2) First component of the adjoint solutions and $(x(t), y(t))$ ((inset), purple and pink lines, respectively) during one cycle of the red canard solutions in panel (b2).

tivity and variability across experimental trials, usually attributed to the network noise, despite the same value of the input control. Several causal effects can be invoked but one which has not received much attention is the canard phenomenon, and it can explain these features. Maximal canard solutions offer the best approximation to excitability thresholds ${ }^{23,35}$, canards in general mediate transitions between complex patterns ${ }^{36}$, and they are sensitive to initial conditions without relying upon chaos.

A key point worth noticing is that even though canard cycles exist only within very narrow parameter ranges, canard segments occur transiently when the system operates in the excitable regime. These key features may give the impression that canards are "rare" events, and that it is hopeless to expect them to be relevant in the experimental world. However, against the common sense, the following examples justify canards as a central mechanism to explain experimental observations. Canard segments have been observed in excitable electronic circuits $^{37}$, which can be seen as more easily-controllable low-dimensional representations of the excitability in real neurons. Another experimental observation on the effects of slow manifolds has been done in the oscillating Belouzov-Zhabotinsky reaction under batch conditions ${ }^{38}$. Moreover, it has been theoretically and computationally- evidenced that canards act as boundaries between different vastly-separated activity regimes. For example, in neurons, they can explain the transition between resting state, spiking, bursting and other electrical activities. Canard-like segments can be employed to interpret single cells' electrophysiological recordings of the membrane electrical potential as activity epochs where the potential slowly evolves near threshold. During such epoch, two scenarios can occur: (1) The neuronal membrane can trigger an action potential and thus can be interpreted as canard with head trajectories that have crossed the threshold (maximal canard). (2) In the opposite case where the membrane fails to trigger an action potential. This can be seen as canard without head trajectories that are unable to cross the threshold. In short, the transition between different neuronal electrical activities can be thought of as manifestations of canard-like behaviors.

\section{E. Reduction of system (2) to an ODE}

The aforementioned anticipation phenomenon setting gives rise to a delay differential equation (DDE), which is notoriously hard to analyse given the high dimensionality of the system. However, under the assumption of 
small delay $\tau$, it is possible to apply a Taylor expansion that reduces the DDE to an ordinary differential equation (ODE) making it more amenable to analysis. We refer the reader to the mathematical conditions under which this approximation is valid in Ref 39. In the case of one variable with only a delayed coupling (e.g. VDP), Taylor expanding the one-dimensional delayed signal $x(t-\tau)$ gives:

$$
x(t-\tau) \approx x(t)-\tau \dot{x}(t)+\frac{1}{2} \tau^{2} \ddot{x}(t)+O\left(\tau^{3}\right),
$$

where $O\left(\tau^{3}\right)$ represents higher-order terms. This methodology has been applied to study canard cycles in delayed slow-fast systems and demonstrate delayinduced canard explosions ${ }^{40,41}$. The technique has also been applied in experimental studies of radiofrequency electronic oscillators to obtain lag and anticipated synchronisation ${ }^{18}$. Similarly, in Ref. 42 a firstorder approximation was used to renormalise the receiver's timescale and study the sender-receiver interaction. In particular, the authors discussed that a positive delay yields anticipation whereas negative delay yields lagged behavior. We will use the same reduction, but contrary to the argument in Ref. 42 we will show that a positive delay can lead to lagged behavior in the canard regime.

\section{RESULTS}

We now apply the aforementioned methodologies to two examples of excitable systems; one from electronics engineering (VDP) and the second a neuronal system $(\mathrm{HH})$. Towards the end of this section, we will propose an experimental protocol to observe anticipation in real neurons and thus validate the predictions made by our theoretical and computational framework.

\section{A. Canard-mediated anticipation in coupled VDP systems}

Following Ref. 4, we consider a system of delayed coupled VDP systems. However we note that in Ref. 4 the case of two unidirectionally-coupled systems was analyzed, with the receiver subjected to a self-feedback loop. Therein it was found that the responses of two coupled systems receiving the same pulse input can be different. Depending on the coupling/feedback strength the two systems can oscillate in synchrony, exhibiting either anticipated or delayed synchronization, or the receiver can oscillate more often than the sender, due to the presence of the feedback loop. In what follows, we drop the self feedback and only consider the typical setting for anticipation described in Eq. (2). The equations consist of two unidirectionally delayed coupled VDP systems receiving a pulse input and reads as follows:

$$
\begin{aligned}
& \dot{x_{1}}=y_{1}-\frac{x_{1}^{3}}{3}+x_{1}+p P(t), \\
& \dot{y_{1}}=\varepsilon\left(c-x_{1}\right), \\
& \dot{x_{2}}=y_{2}-\frac{x_{2}^{3}}{3}+x_{2}+\alpha\left(x_{1}-x_{2, \tau}\right)+p P(t), \\
& \dot{y_{2}}=\varepsilon\left(c-x_{2}\right),
\end{aligned}
$$

where $\left(x_{1}, y_{1}\right)$ represents the sender, $\left(x_{2}, y_{2}\right)$ the receiver and $p$ is the amplitude of the external pulse $P(t)$. In order to identify a possible relation between different spiking properties of (7) and canards, we approximate the delay term $x_{2, \tau}$ with the first-order terms of its Taylor expansion (6), assuming that the time delay $\tau$ is sufficiently small. This leads to the following reduced ODE system:

$$
\begin{aligned}
\dot{x_{1}=} & y_{1}-\frac{x_{1}^{3}}{3}+x_{1}+p P(t) \\
\dot{y_{1}=} & \varepsilon\left(c-x_{1}\right) \\
\dot{x_{2}=} & \frac{1}{1-\tau \alpha}\left(y_{2}-\frac{x_{2}^{3}}{3}+x_{2}+\alpha\left(x_{1}-x_{2}\right)+\ldots\right. \\
& \left.\ldots p P(t)+O\left(\alpha \tau^{2}\right)\right) \\
\dot{y_{2}=} & \varepsilon\left(c-x_{2}\right) .
\end{aligned}
$$

Both the Taylor expansion of the delayed term and the coupling strength $\alpha$ introduce higher-order terms, $O\left(\alpha \tau^{2}\right)$; it also modifies the timescale of the fast component of the receiver by $\tau_{\alpha}:=(1-\tau \alpha)^{-1}$; see Ref. 42 . We will primarily consider small delay and weak coupling strength, so that the first-order Taylor expansion is justified and consequently weak-coupling theory applies. Moreover, we will only keep the first-order approximation, thus we drop the $O\left(\alpha \tau^{2}\right)$ term. Higher-order terms could potentially be considered for a more refined analysis but this is beyond the scope of the present work. To test the robustness and validity limits of the proposed framework we will increase both $\tau$ and $\alpha$, and numerically verify whether the "small delay/ weak coupling" approximation persist far away from these double limits.

The additional timescale, $\tau_{\alpha}$, is a key element that makes anticipation possible. When both sender and receiver systems are initialized at their common equilibrium point, then at the precise time when the external pulse is applied, the only difference between the two systems is through $\tau_{\alpha}$. This parameter is slightly greater than 1 for small values of $\tau$ and $\alpha$, which makes the receiver slightly faster than the sender at the moment of the pulse initiation. If the pulse is strong enough to drive both systems beyond the spiking threshold, this has the effect to "kick" the receiver further away from the critical manifold than it does to the sender. Therefore, the receiver with a faster speed crosses the threshold before the sender does. For the configuration we study here, this explains why anticipation occurs. However, we need further insights in order to identify the boundary of the 
anticipatory regime, which is related to maximal canards since they give the best approximation of the excitability threshold (in these models) ${ }^{23}$.

In order to understand how anticipation depends upon parameters such as the amplitude of the external forcing $p$ or the delay $\tau$, we need a precise numerical strategy that allows us to deal with the sensitivity introduced by the slow-fast nature of the problem. To this end, we use a numerical continuation scheme for solving twopoint boundary-value problems (BVPs) with the software package $\mathrm{AUTO}^{43}$. However, two adjustments must be made to compute the solutions of this system close to the boundary of the anticipation regime. First, we replace the pulse (which is active between $t_{\text {on }}$ and $t_{\text {off }}$ ) by a steep-enough sigmoid function to ensure smoothness of the solutions of the numerical problem, namely:

$$
P(t)=\frac{1}{1+\mathrm{e}^{k\left(t-t_{\mathrm{on}}\right)}}-\frac{1}{1+\mathrm{e}^{k\left(t-t_{\text {off }}\right)}} .
$$

Second, we add the trivial equation $\dot{t}=1$ to the system (8) so that it becomes autonomous, which is essential for the numerical continuation procedure to work. Hence we obtain a five-dimensional extended system. The main goal is to understand transient responses of the original system (8), from an equilibrium, to the pulse perturbation given by equation (9) upon parameter variation. To this end, we consider a two-point BVP on the extended system and we seek a one-parameter family (in our case, c) of solutions segments that start from equilibrium, undergo the perturbation and settle back to the original equilibrium. A key point of interest is to compute the trajectory time, that is the integration time $T_{c}$ for the entire one-parameter family of solutions. This time can then be associated with the period function of the system. Therefore, we set the two-point BVP on this extended five-dimensional system as follows. We first impose that the initial conditions of both sender and receiver are $\left(x_{\text {rest }}, y_{\text {rest }}\right)$. Indeed, for any $|c|>c_{H}$, both the sender and the receiver's equilibrium state is given by $\left(x_{\text {rest }}, y_{\text {rest }}\right)=\left(c, \frac{c^{3}}{3}-c\right)$. Furthermore, when varying the system parameter $c$, the resulting one-parameter family of solution segments will have different associated integration times $\left(T_{c}\right)$. Therefore, $T_{c}$ will be free to vary within the BVP setup, and its associated a priori unknown value will be found at each step as the BVP is solved. However, to enable the variation of two quantities $\left(c\right.$ and $\left.T_{c}\right)$ in a five-dimensional system, six boundary conditions are required so that the problem is numerically well-posed and possesses a unique solution at every continuation step. We therefore impose the following six boundary conditions:

$$
\begin{aligned}
x_{1}(0) & =x_{\text {rest }}, \\
y_{1}(0) & =y_{\text {rest }}, \\
x_{2}(0) & =x_{\text {rest }}, \\
y_{2}(0) & =y_{\text {rest }}, \\
t(0) & =0, \\
x_{1}(1) & =x_{\text {rest }} .
\end{aligned}
$$

Note that $T_{c}$ becomes an unknown of the numerical problem via an appropriate time rescaling $\left(t \mapsto t / T_{c}\right)$, which recasts an autonomous $\operatorname{ODE}(\dot{x}=\mathbf{F}(x, c))$ to the following rescaled ODE $\left(x^{\prime}=T_{c} \mathbf{F}(x, c)\right)$ and also has the effect that the boundary conditions are taken at $t=0$ and $t=1$. This time rescaling also allows us to compare the integration time of the computed orbits with $T_{\varepsilon}$. The six boundary conditions given above are composed by five initial conditions for both the sender and the receiver, as well as, time $t$, which is a variable in this extended problem, together with one end condition. The last boundary condition is defined in order to make the overall problem numerically well-posed. Given that we have a 5 dimensional problem with an unknown integration time, $T_{c}$, and 6 boundary conditions (10), hence at each step of the computation, a unique solution is guaranteed since we have equal number of constraints and unknowns. Then, if we vary an additional free parameter, which can be either $\alpha, \tau$ or $p$, we can compute a one-parameter family of solution segments. In brief, the above boundary conditions permit to measure the time required for the system to return to the rest state after being perturbed away from it. The unknown quantity $T_{c}$ by the continuation procedure can then be considered as the transient response time to the perturbation.

\section{B. Pulse-driven canard trajectories}

Baer and Erneux ${ }^{44,45}$ showed that perturbations of the Hopf bifurcation parameter in a slow-fast ODE $(c$ in system (8)) lead to canard transitions. Campbell and colleagues ${ }^{40}$ extended these results to a system for which a small delay introduces a certain perturbation and showed, using perturbation methods and Chicone's results $^{39}$ on small-delay approximation, that a supercritical Hopf bifurcation persists, followed by a canard explosion. This justifies our setup. Since we want to keep the framework of Ref. 4, we consider the pulse perturbation in (8) for a fixed $c$ value. We initialize the system at a rest state in the absence of coupling and delay $(\alpha=0, \tau=0)$ and track, using the BVP (10), the pulse-driven trajectories under the variation of the pulse amplitude $p$.

In Fig 3 we compute the response of the sender $\left(x_{1}, y_{1}\right)$ initialized at $c=1.01$ for $\varepsilon=0.09$. On panel (a) $T_{c}$ is given as a function of the pulse amplitude. The behavior of $T_{c}$ is similar to the non-monotonic behavior of the period function of the canard explosion (Fig 2). As $p$ increases, $T_{c}$ increases sharply until it reaches a maximum value and then it decreases. This indicates that $\left(x_{1}, y_{1}\right)$ undergoes a canard explosion as a function of $p$.

The trajectories along the stiff curve $T_{c}$ as a function of $p$ are the sub-threshold solutions, which follow the right attracting branch and the repelling middle branch of the critical manifold. The blue and green trajectories, shown in panels (b) and (c) of Fig 3, belong to this class. The red trajectory on the middle panel is the solution with the maximal value of $T_{c}$ and it has the longest repelling 

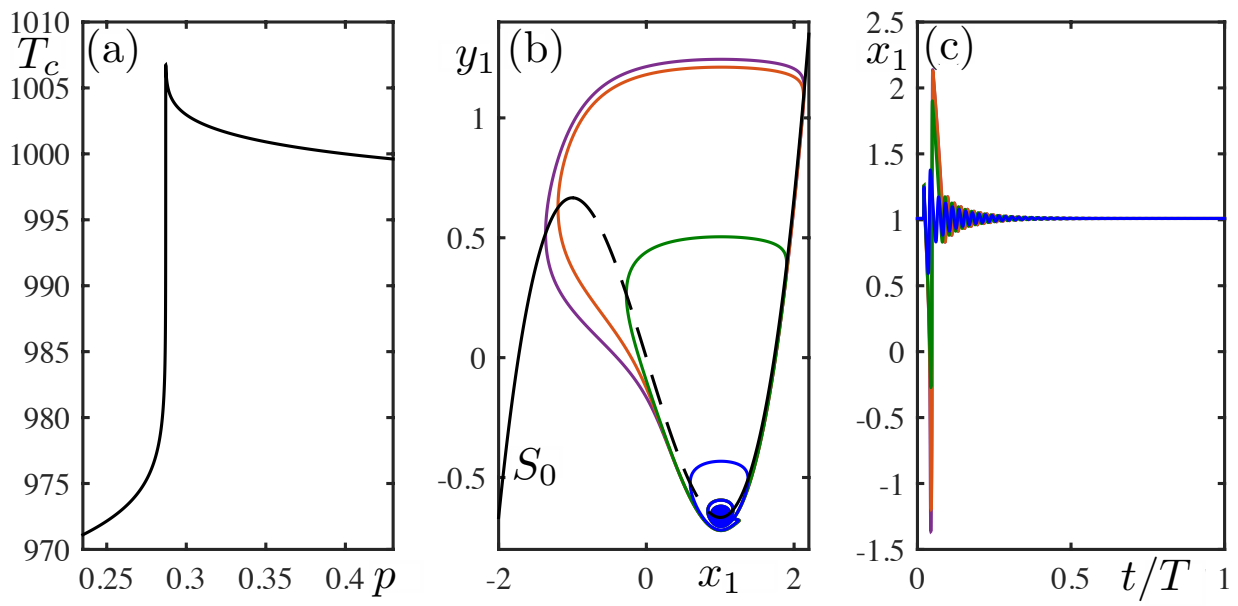

FIG. 3. Response of the sender to pulse perturbation in (8): (a) Integration time $T_{c}$ vs pulse amplitude $p$ for $\varepsilon=0.09$. Increasing pulse amplitude results in a transition form small canard trajectories to large canard trajectories. (b) Phase portrait for four different solutions: $p \approx 0.27141141457$ (blue), $p \approx 0.28705449402$ (green), $p \approx 0.28710623834$ (red), $p \approx 0.28723689613$ (purple). The black curve is the critical manifold $S_{0}$ with attracting (solid) and repelling (dashed) branches. (c) Time trace of the $x_{1}$-component of the solutions given in panel (b).

segment. This solution can be considered as the maximal canard and it establishes, as explained before, the boundary between a spiking and a non-spiking behavior. For larger values of $p$ the solutions enter into the spiking regime by jumping off the repelling branch towards the left attracting branch (purple trajectory in panels (b) and $(c))$. Since the solutions in the spiking regime have a shorter segment along the repelling branch as $p$ increases, $T_{c}$ decreases.

\section{Effect of the delayed coupling}

The analysis given above shows that when the sender is initialized at the resting state, it can be driven towards the repelling slow manifolds by a pulse perturbation. The length of the canard segment, the duration of the transient response, and the type of resulting solutions depend on the amplitude of the perturbation. In this subsection, we investigate the effects of $\tau$ and $\alpha$ system (8) for different values of $p$.

In Fig. 4 both $\alpha$ and $\tau$ are fixed to relatively small values. On panels (a1) and (b1), we start from equivalent sub-threshold solutions for the sender and the receiver with set values, $p \approx 0.28705492172, \alpha=0.001$ and $\tau=0$, and apply continuation procedure to these systems with $\tau$ as main continuation parameter. As $\tau$ increases, the coupling term drives the receiver towards the upper fold. Hence, the receiver with longer canard segment stays longer along the repelling branch. In this case the receiver jumps to the right branch after the sender does and consequently it is delayed. This behavior is coherent with the adjoint solution, $Z_{1}(t)$, for the small canard where a weak positive perturbation near the right of the lower fold delays the phase of a headless canard cycle.

In Fig. 4 (a2) and (b2) we fix $p \approx 0.28723689613, \alpha=$ 0.01 and we investigate the effect of the delayed coupling on the spiking solutions. In contrast to the sub-threshold solutions, the delayed coupling in the receiver shortens the length of its canard segment. Hence, the receiver leaves the repelling middle branch of $S_{0}$ earlier than the sender. This behavior results in anticipation, which is consistent with the evolution of the adjoint solution $Z_{1}(t)$ for large canards, where perturbations to the right of the lower fold advance the phase of a canard-with-head cycle. Note that spiking solutions are less sensitive to perturbations than non-spiking ones, thus the $\alpha$-values that give a substantial anticipation are larger than those corresponding to the right-panel solutions.

The simple analysis shown in Fig. 4 indicates that delayed unidirectional interactions do not yield anticipation for the entire family of trajectories of an excitable system. Essentially, the type of trajectory and the length of its canard segment determine the impact of the coupling. When the pulse perturbation and suitable coupling maintain trajectories below threshold, the receiver system is delayed with respect of the sender. In contrast, the receiver anticipates the sender when both subsystems belong to the canard-with-head family.

In Figs. 5 and 6 we increase both $\alpha$ and $\tau$ beyond the limit of the weak-coupling and small-delay approximation. The weak-coupling theory summarised previously does not apply and the error due to the firstorder approximation is large for these parameter regions. Including higher-order terms in the expansion reduces the quantitative error between the solutions of the DDE and those of the ODE but the qualitative behavior remains the same (data not shown). We track the dif- 
(a1)

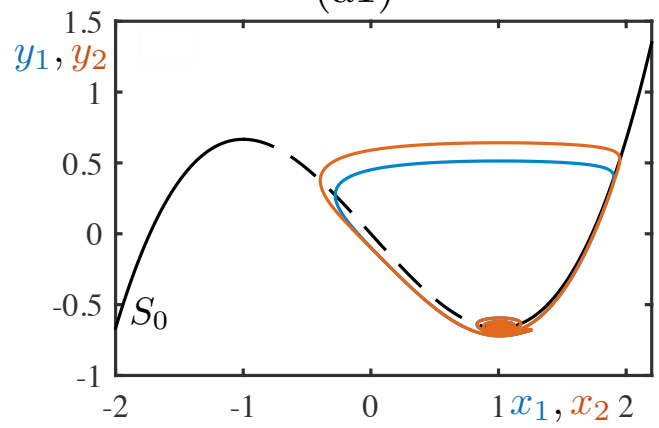

(b1)

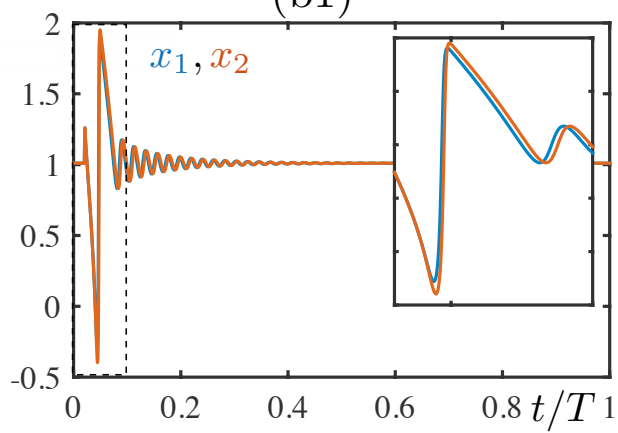

(a2)

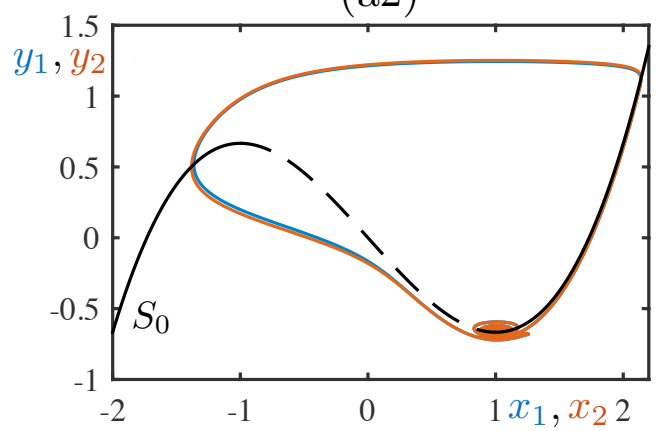

(b2)

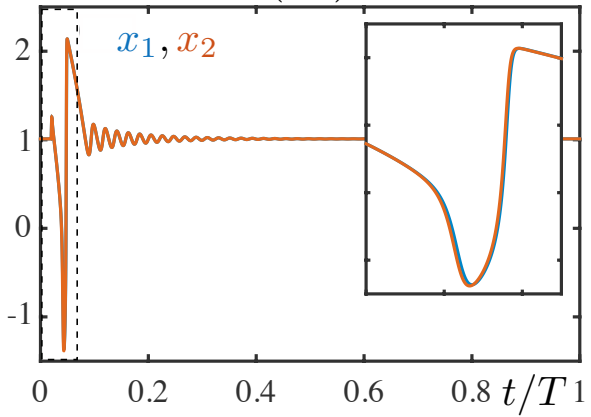

FIG. 4. Example solutions of system (8) under pulse perturbation: Lower panels are the time series of some solutions represented in the upper panels. The black curves on (a1) and (a2) are the critical manifolds $S_{0}$ with attracting (solid) and repelling (dashed) branches.. The dashed boxes on (b1) and (b2) are zoomed in the insets. (a1)-(b1) The sender (blue) and receiver (red) are below threshold for $p \approx 0.28705492172$. (a1) The receiver $(\alpha=0.001, \tau=0.01$ ) has a larger canard segment than the sender (blue). (b1) Receiver (red) tracks the sender (blue). (a2)-(b2) The sender and receiver are above threshold for $p \approx 0.28723689613$. (a2) The receiver (red, $\alpha=0.01, \tau=0.01$ ) has a larger canard segment than the sender (blue). (b2) The receiver anticipates the sender.

ference between the minimum values of the fast variables $\left(\min \left(x_{1}\right)-\min \left(x_{2}\right)\right)$ so as to visualize the discrepancy between the sender and the receiver. The explosive diagram obtained for a small sub-threshold solution on Fig. 5 (a) shows that the receiver can move from the non-spiking regime to the spiking regime by following repelling slow manifolds as $\tau$ increases. The right panels show an example solution of this extreme case where the gap between two systems is very pronounced: the receiver spikes while the sender remains silent.

Trajectories that already start in the spiking regime do not undergo any canard-explosive transition; see Fig. 6. Still, the perturbation on the receiver shortens the length of its canard segment but the discrepancy between the two systems increases. The difference $\left(\min \left(x_{1}\right)-\min \left(x_{2}\right)\right)$ increases because the canard segment of the receiver system is always shorter than that of the sender. Consequently, a perturbation of the solutions in this region causes the receiver to anticipate the sender. The inset in Fig. 6 (b1) clearly shows the effect of a "kick" on the receiver, that is to push it further away from the critical manifold than it does to the sender.

Next, we extend our observations from pulse perturbation to periodic solutions of (8) for $p=0$. For this, we consider $\alpha$ and $\tau$ values smaller than our previous anal- ysis in the transient regime to avoid higher-order harmonics. Figure 7 presents there different regimes. For the subthreshold regime in panels (a1) and (a2), the receiver is delayed because it follows the repelling branch of $S_{0}$ longer than the sender. For a subthreshold setting closer to the maximal canard in panels (b1) and (b2), the receiver is driven above the threshold while the sender oscillates below the threshold. Panels (c1) and (c2) illustrate that the system for spiking solutions maintains the AS regime. This analysis shows that the role of attracting and repelling slow manifolds on ancitipation can be preserved in the periodic regime. The effects of higherorder harmonics for the parameters beyond the limits of weak coupling and small delay terms deserve further investigations, especially on the subthreshold solutions.

\section{Anticipation via canards in coupled 2D $\mathrm{HH}$ systems}

The anticipation phenomenon also emerges in neuronal models. An immediate observation of this is to notice that the FitzHugh-Nagumo model, which is a well-known model of excitable system, is in fact a slightly modified version of the VDP system. Specifically, the difference between the VDP and FHN systems is that the 
(a)

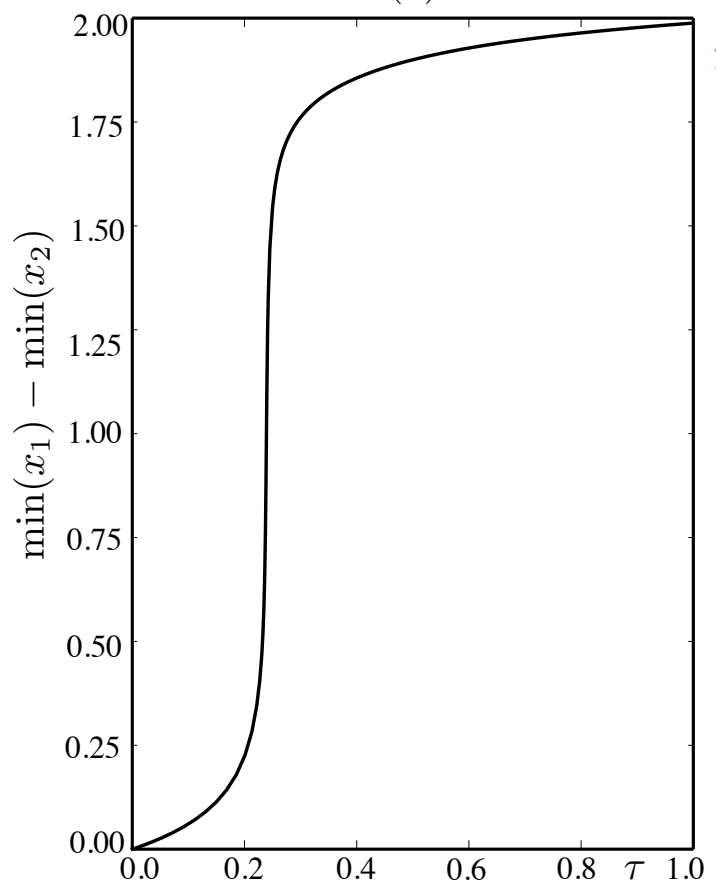

(b1)

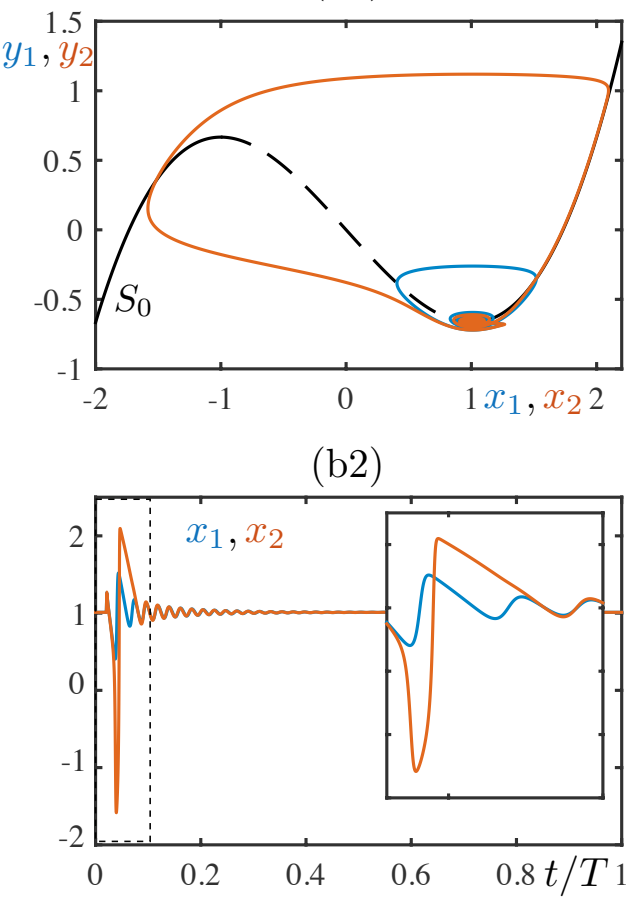

FIG. 5. Behavior of system (8) under pulse perturbation for $p \approx 0.27141141, \alpha=0.05, \tau \in[0,1]:(\mathrm{a}) \min \left(x_{1}\right)-\min \left(x_{2}\right)$ vs $\tau \in[0,1]$. (b1) Projection of the sender (blue) and receiver (red) at $\tau=1$ on the phase plane. The black curve is $S_{0}$ with attracting (solid) and repelling (dashed) branches. (b2) Time series of the solution on (b1) where the sender remains below the threshold while the receiver spikes. The inset zooms into the dashed box on the panel (b2).

slow linear dynamics of FHN has a term in $y$ whereas it does not for VDP. A non-spiking solution corresponds to a small canard trajectory which passes near the lower fold $\left(x_{f}^{+}, y_{f}^{+}\right)$and a spiking solution is a trajectory which passes near both the lower fold $\left(x_{f}^{+}, y_{f}^{+}\right)$and the upper fold $\left(x_{f}^{-}, y_{f}^{-}\right)$. Therefore, anticipation is expected in the FHN model. Besides, to further illustrate our framework for explaining anticipation in neuronal system we will focus on a more biophysical model of neuronal excitability, namely a 2D reduction of the classical $\mathrm{HH}$ model, which has been analyzed from the viewpoint of canard dynamics by Moehlis ${ }^{46}$. The purpose of this example is to show that the 2D HH model retains anticipation via canards, that is at the level of transient dynamics, but also AS via canards, which is an asymptotic phenomenon. We expect that the same anticipation predictions can be made for higher-dimensional neuronal models. This 2D reduced $\mathrm{HH}$ model is written as follows:

$$
\begin{aligned}
C \dot{V}= & I-\bar{g}_{N a}\left[m_{\infty}(V)\right]^{3}(0.8-n)\left(V-V_{N a}\right)-\ldots \\
& \ldots \bar{g}_{K} n^{4}\left(V-V_{K}\right)-g_{L}\left(V-V_{L}\right) \\
\dot{n}= & \alpha_{n}(V)(1-n)-\beta_{n}(V) n,
\end{aligned}
$$

where $V$ represents membrane potential, $n$ represents the activation of potassium channels, and the functions are $\alpha_{n}(V)=(0.01(V+55)) /(1-\exp [-(V+55) / 10])$, $\beta_{n}(V)=0.125 \exp [-(V+65) / 80], m_{\infty}(V)=\alpha_{m} /\left(\alpha_{m}+\right.$ $\left.\beta_{m}\right)$ with $\alpha_{m}=(0.1(V+40)) /(1-\exp [-(V+40) / 10])$, $\left.\beta_{m}=0.4 \exp [-(V+65) / 18]\right)$.

Moehlis studied system (11) with the following fixed values of the parameters: $\bar{g}_{N a}=120, \bar{g}_{K}=36, \bar{g}_{L}=0.3$, $V_{N a}=50, V_{K}=-77, V_{L}=-54.4, C=1$, leaving $I$ as the main bifurcation parameter. After showing numerically that the dynamics of $V$ is much faster than that of $n$, hence justifying that the system exhibits slow-fast dynamics, Moehlis performed a formal asymptotic analysis in $\varepsilon$. This, allowed to rescale the system and explicitly write the evolution of the $n$ variable as a slow process. Additionally, an $\varepsilon$-expansion was obtained for the $I$-value at which the canard explosion occurs.

Fig. 8 shows the behavior of system (11) for $I=6.35$ and subject to the pulse perturbation $p P(t)$ on the fast variable. On panel (a) we plot the amplitude of the variable $V$ of the sender as a function of the pulse amplitude $p$. For weak perturbations $(p \in(0,2.5))$, the system remains in a small neighborhood of the rest state. As $p$ increases, the trajectories start to follow the repelling branch of the critical manifold and the solutions grow in amplitude. For $p \approx 2.5856531235$ the solution remains close to the entire repelling branch of $S_{0}$, hence this trajectory contains a maximal canard segment for the pulse-perturbed systems. Once the trajectories enter the canard-with-head region, corresponding to the spiking regime, then $T_{c}$ decreases although the solutions continue to grow in amplitude. Fig 8 (b) and (c) show the phase-plane projections and time profiles for some of 
(a)

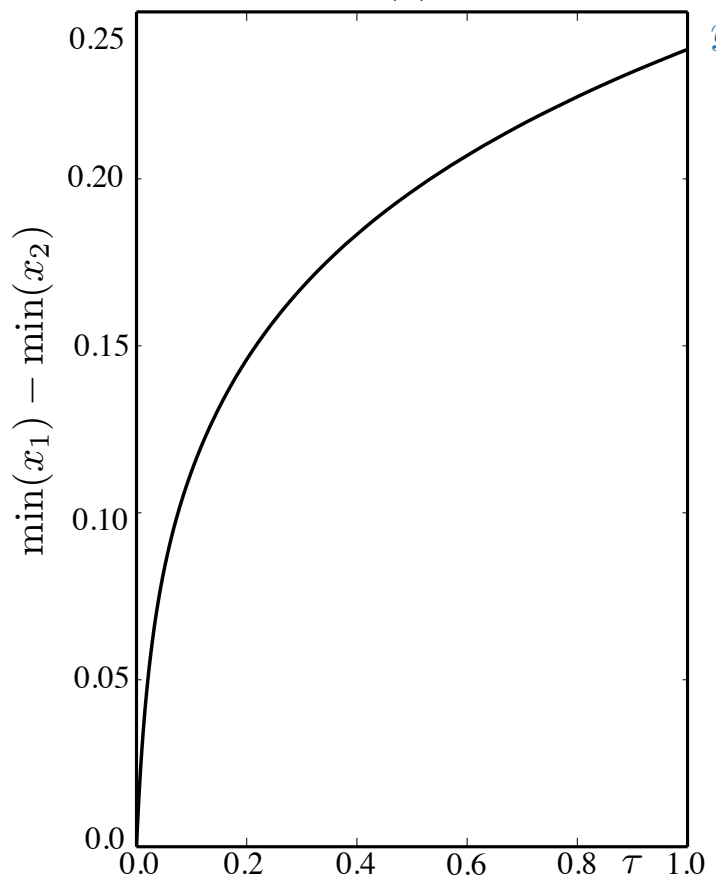

(b1)

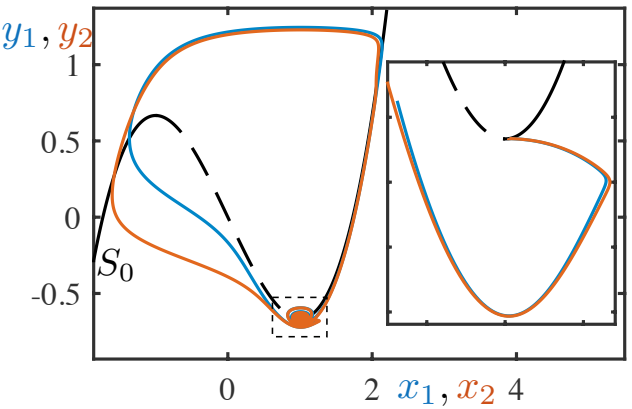

(b2)

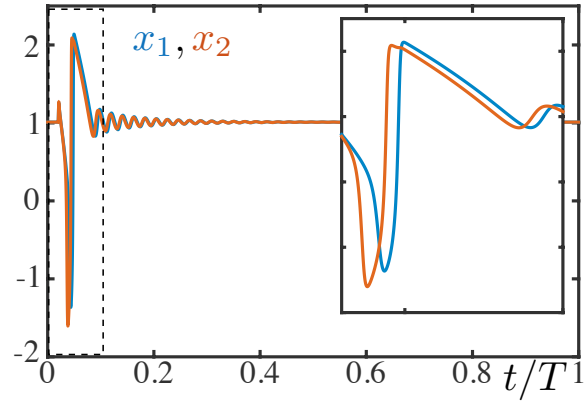

FIG. 6. Behavior of system (8) under pulse perturbation for $p \approx 0.28723689613, \alpha=0.05, \tau \in[0,1]:(\mathrm{a}) \min \left(x_{1}\right)-\min \left(x_{2}\right)$ vs $\tau \in[0,1]$. (b1) Projection of the sender (blue) and receiver (red) at $\tau=1$ on the phase plane. The black curve is $S_{0}$ with attracting (solid) and repelling (dashed) branches. The inset in (b1) shows a part of the solution just after the kick. The receiver receives a stronger kick due to $\tau_{\alpha}$ and it moves faster than the sender. (b2) Time series of the solution on (b1) where the sender spikes after the receiver The inset zooms into the dashed box on the panel (b2).

these canard solutions.

We now couple two identical 2D HH systems (11), subject to a pulse input in each fast variables. A unidirectional coupling from the sender $\left(V_{1}, n_{1}\right)$ to the receiver $\left(V_{2}, n_{2}\right)$ as well as a delayed feedback term in the re- ceiver are included, similar to what we did for the VDP systems in (7). The coupling term is hence of the form $\alpha\left(V_{1}-V_{2, \tau}\right)$. As in the VDP example, we replace the delayed signal by its first order Taylor expansion, that is, $V_{2, \tau} \approx V_{2}-\tau \dot{V}_{2}$. Therefore, the equations of the unidirectionally delayed-coupled systems are given by:

$$
\begin{aligned}
C \dot{V}_{1} & =I+p P(t)-\bar{g}_{N a}\left[m_{\infty}\left(V_{1}\right)\right]^{3}\left(0.8-n_{1}\right)\left(V_{1}-V_{N a}\right)-\bar{g}_{K} n^{4}\left(V_{1}-V_{K}\right)-g_{L}\left(V_{1}-V_{L}\right) \\
\dot{n_{1}} & =\alpha_{n}\left(V_{1}\right)\left(1-n_{1}\right)-\beta_{n}\left(V_{1}\right) n_{1} \\
(C-\alpha \tau) \dot{V}_{2} & =I+p P(t)-\bar{g}_{N a}\left[m_{\infty}\left(V_{2}\right)\right]^{3}\left(0.8-n_{2}\right)\left(V_{2}-V_{N a}\right)-\bar{g}_{K} n^{4}\left(V_{2}-V_{K}\right)-g_{L}\left(V_{2}-V_{L}\right)+\alpha\left(V_{1}-V_{2}\right) \\
\dot{n_{2}} & =\alpha_{n}\left(V_{2}\right)\left(1-n_{2}\right)-\beta_{n}\left(V_{2}\right) n_{2} .
\end{aligned}
$$

The behavior of (12) for different values of the pulse amplitude $p$ and the delay parameter $\tau$ is very similar to that of system (8). In Fig. 9 we give some examples of possible solutions. When the sender and the receiver operate under the threshold, a weak perturbation delays the receiver (panel (a)) by increasing the length of the canard segment, while it can move to the spiking region under strong enough perturbations (panel (b)). The receiver anticipates the sender when both operate in the spiking regime (panel (c)), since the sender has a longer canard segment then the receiver.

A pulse perturbation in the unidirectionally delayedcoupled HH system given by (12) can also initiate sustained oscillations. Fig. 10 shows an example for $g_{N a}=$ 122 where the coupled system is driven into AS by the pulse perturbation. This example illustrates possible effects of varying sodium channels' dynamics in real neurons, where a transition from synchronous to AS solutions can be expected. Hence this provides evidence that canards are also important in AS in coupled excitable 

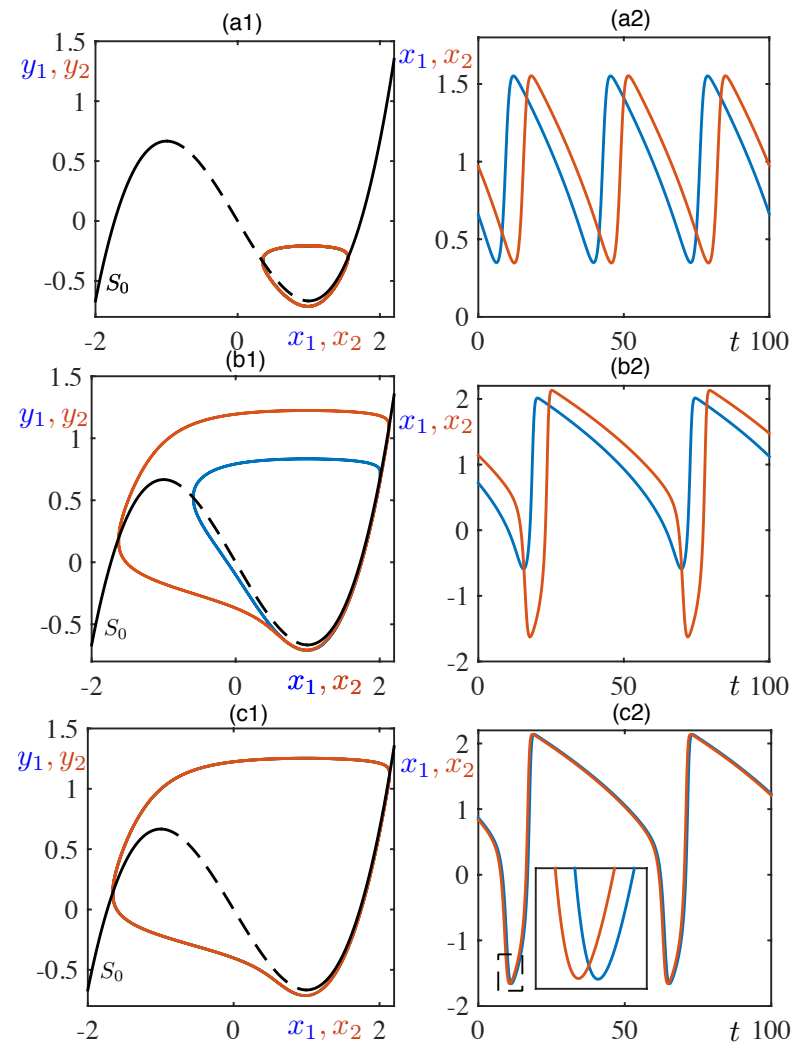

FIG. 7. Stable periodic solutions of system (8) for $p=0$ : Left panels display the phase plane. Right panels display the time series. (a) The slave is delayed for $a=0.988, k=0.001, \tau=$ 0.05. (b) The slave spikes for $a \approx 0.987818, k=0.01, \tau=$ 0.01. (c) The slave anticipates for $a=0.985, k=0.01, \tau=$ 0.5 . The inset in (c2) clearly shows that the slave spikes before the master.

\section{systems.}

Moehlis ${ }^{46}$ found some orbits of the $4 \mathrm{D}$ model which, when projected onto the $(V, n)$-plane, seem similar to canard cycles. Some of these orbits, like in the $2 \mathrm{D}$ reduction, are unstable. We have investigated the same pulse-coupling setting in the coupled 4D models. Direct simulations with XPPAUT ${ }^{47}$ predict that the receiver can follow the unstable branch of the critical manifold for certain $\tau$ and $p$ values; see Fig. 11. Even though these trajectories are unstable, they may have considerable influence on the global dynamics, for instance, a perturbation can drive the systems towards the vicinity of such trajectories and introduce a substantial difference in the output.

\section{E. Towards observing anticipation in neural experiments}

To validate the predictions advanced by our framework, we propose a lab experiment applied to neuronal cells. In the interest of simplifying the complexity of the experiment, we will avoid long-range axonal connections and chemical synapses, which induce delay. Instead, we will take advantage of the fact that one key step in our work to unmasking the anticipation phenomena was the reduction of the sender-receiver system from a DDE to an ODE. In this setting, we note that the delayed coupling in the DDE, is reduced in the ODE to a diffusive term resembling a gap junction. Moreover, this reduction induces an extra timescale separation which depends on the time delay $\tau$. Following the above framework and considerations, we then seek to mimic our results in an experimental controlled setting that also allows the reproducibility of the results across trials.

To this end, we invoke the dynamical-clamp electrophysiological technique, which robustly allows a two-way communication between a computer and neuronal cells in real-time ${ }^{48,49}$. This setup enables high-precision control of neurons, whereby the experimenter has the possibility of studying independent components of the neuron and subsequently testing and validating different model assumptions. For example, in the cellular membrane a particular ion channel can be pharmacologically blocked. Subsequently a proposed mathematical model of that ion channel, running within the computer, can be tested by injecting the output of the model ion channel into the cellular membrane, which leads to changes of the electrical properties of the membrane. This closed-loop setting allows an iterative approach to model validations and precise understanding of neuronal mechanisms. Certainly, such an experiment may be hard to perform successfully, in particular due to noise handling and sensitivity of canard dynamics. However, recent technological advances could mitigate these potential issues, for instance by using nanoprobes instead of classical electrodes ${ }^{50}$ and stateof-the-art feedback-control strategies ${ }^{51}$.

This electrophysiology paradigm has been used by Refs. 52 and 53 to study the synchronization properties of biological neurons bi-directionally coupled via the so-called dual whole-cell recordings, whereby the coupling is mediated by artificial synapses, i.e. models running in the computer. Interestingly, Wang et.al. ${ }^{53}$ have also studied synchronisation by considering delayed synaptic interaction where the synaptic activity in the post-synaptic cell was delayed by a user-defined value mediated va a computer. In this work, experimental PRC curves of neurons could be measured and estimated. They showed that short delays can lead to bistable modes where the identical neurons can interchange leader/follower roles. Therefore a similar feasible experimental paradigm can be considered to investigate the behavior in canard regimes. However, instead of considering mutually-coupled cells via a chemical synapse, we propose unidirectional coupling between two identical neurons where a gap junction model is mediated by the dynamic-clamp computer; see Fig. 12. A key point is to notice that in our framework the reduction from DDE to ODE induces an extra timescale separation in 
(a)

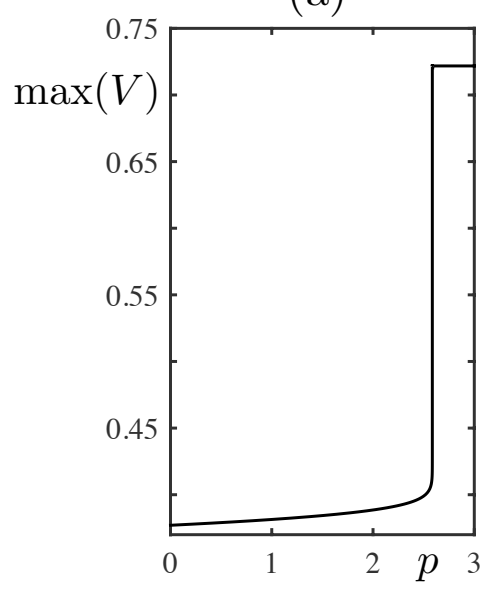

(b)

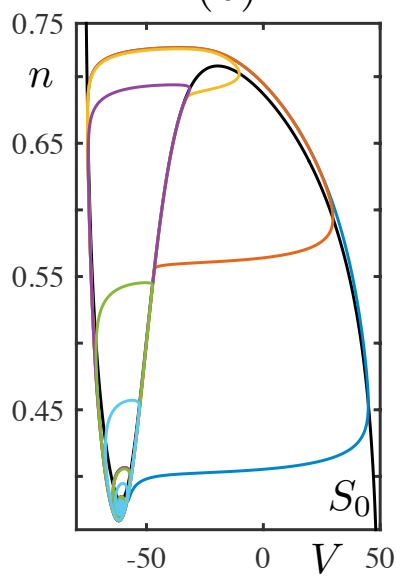

(c)

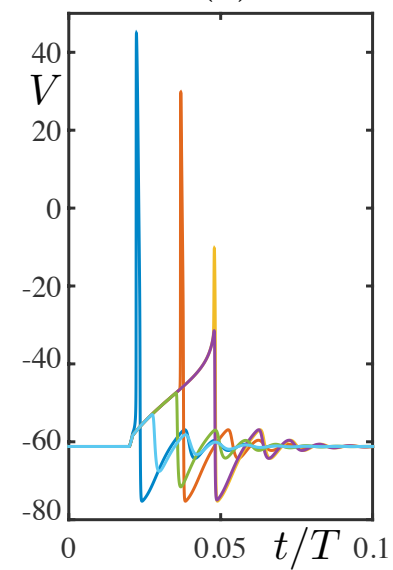

FIG. 8. Response of system (11) receiving a pulse input: (a) $\max (V)$ vs $p$ diagram. Depending on the pulse amplitude $p$, the solution moves along the repelling slow manifold and has a canard segment. (b) Some trajectories in the ( $V, n)$ plane and the critical manifold $S_{0}$. (c) Time traces of the trajectories in panel (b)
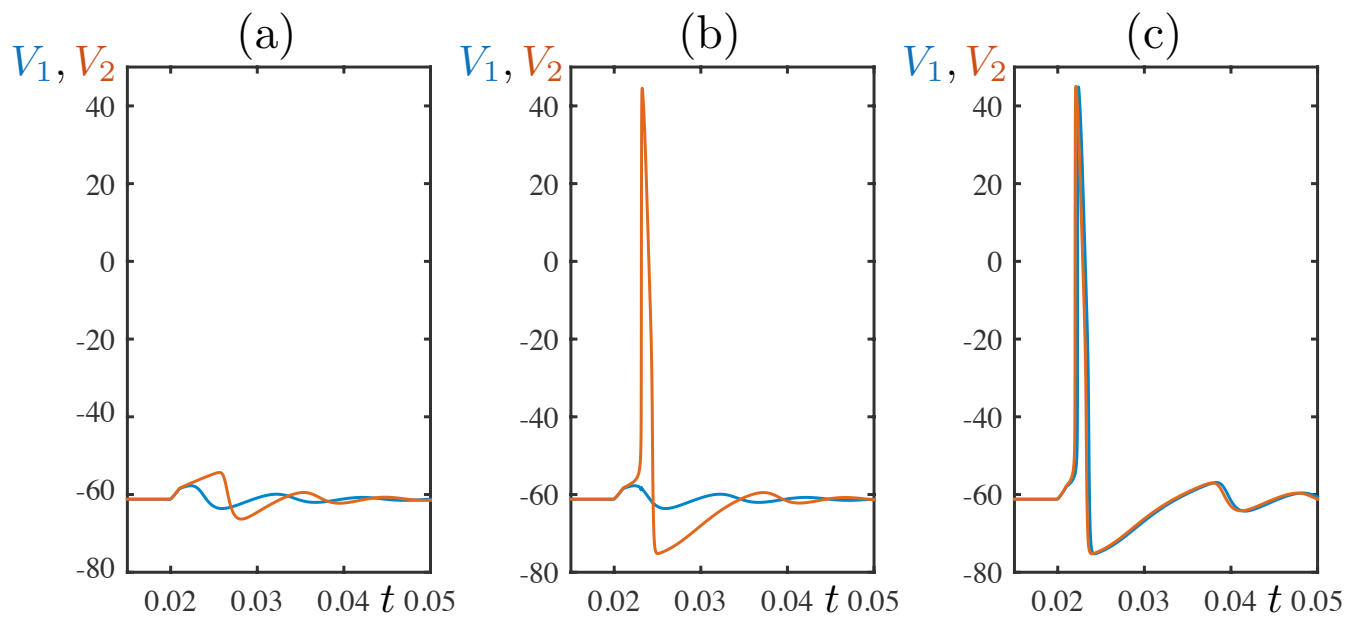

FIG. 9. Example trajectories of the receiver (red) and the sender (blue) in system (12) for $\alpha=0.05$ : (a) Both systems are below threshold for $\tau \approx 0.59752753475, p \approx 2.4955934072$. (b) The receiver spikes while the sender remains below threshold for $\tau \approx 0.92363605436, p \approx 2.4955934072$. (c) The receiver anticipates the sender for $\tau=1, p \approx 2.9196246953$.

the receiver. This implies a further change of the cellular membrane properties of the receiver, which has to be mimicked in the experimental setting. To this end, two sub-experiments could be considered: (1) One experimental protocol would make the assumption that no two cells of the same family are completely identical (i.e. intrinsic variability), which may imply automatically that one cell has an extra timescale in the response of its cellular membrane. However, in this setting we would not have direct control of the extra timescale, but it is possible that AS would be nevertheless observed. (2) The second experimental protocol would ensure that the receiver has indeed an extra timescale, which can be induced or enforced either pharmacologically or possibly genetically. Specifically, the kinetics of the potassium ion channels, in particular their sub-units, associated to the onset of action potentials (spikes) in the receiver cell could be pharmacologically or genetically tuned. Alternatively, the kinetics of the potassium ion channel of the master neuron could be slowed-down. For completeness and precise control, the full experimental setup would actually block chemical synapses and all other currents, except voltage-dependent sodium and potassium channels which would be preserved.

\section{DISCUSSION}

Anticipation apparently violates the principle of causality in mechanistic models of science and engineering. Consequently, the counter-intuitive observation of anticipated synchronization in dynamical systems has be- 
(a)

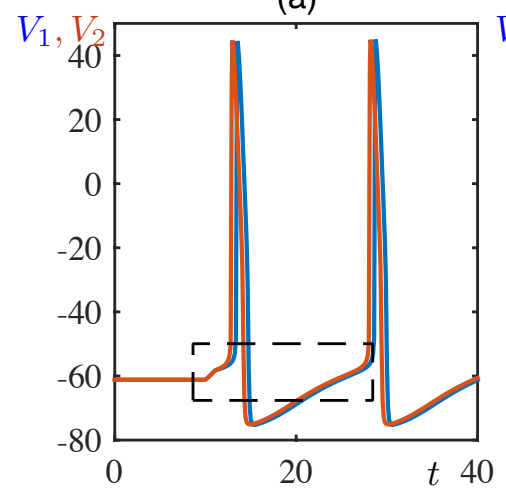

(b)

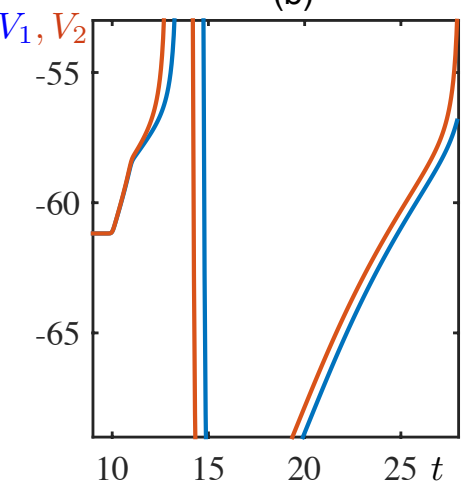

(c)

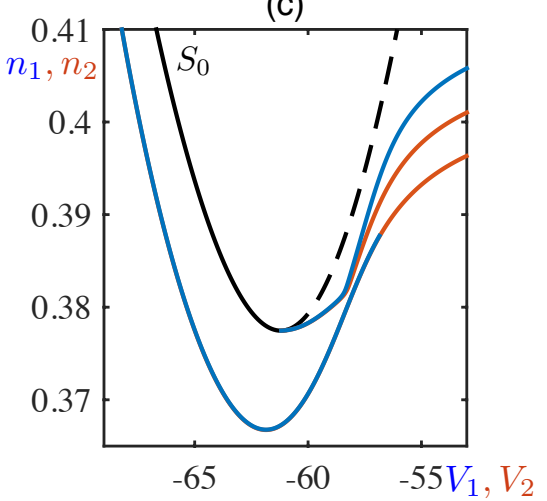

FIG. 10. Anticipated synchronisation in system (12). (a) Anticipated behavior can be initialised for $\bar{g}_{N a}>120$ by a pulse that can drive the coupled system (12) into the oscillatory regime. If the systems are delayed coupled for $\tau>0$, then anticipated synchronous behavior can be observed. $\bar{g}_{N a}=122, \tau=0.5, \alpha=0.05, p=2.5$ (Blue: Sender. Red: Receiver.). Inner box is zoomed in (b) where the amplitude of the receiver is less at $t=27.9190$. (c) Projection of the time series zoomed in (b) on the phase plane near the lower fold of $S_{0}$; the black bold curve corresponds to the attracting (solid) and to the repelling (dashed) parts of $S_{0}$. After being perturbed, the receiver remains close to the repelling branch of $S_{0}$ along a shorted segment than the sender, hence, the receiver anticipates the sender during the next cycle.

(a)

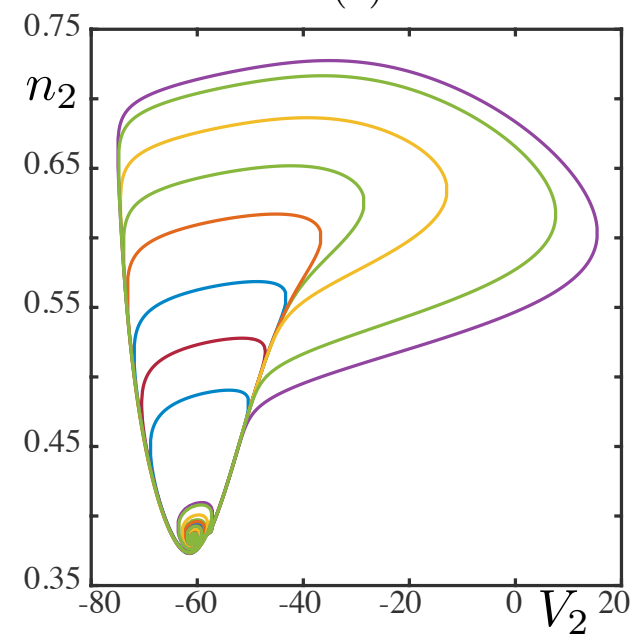

(b1)
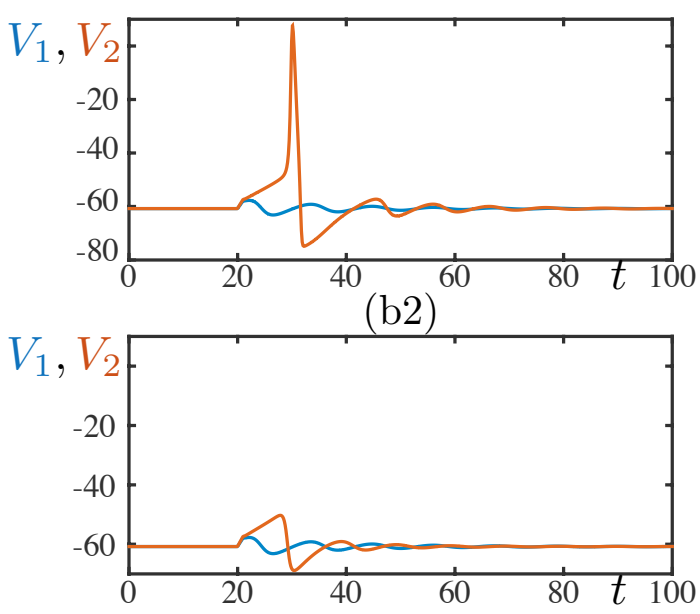

FIG. 11. Several solutions of the receiver in the full (4D) HH system for $\alpha=0.05, \tau=4$ and $p$ varied in the vicinity of $p \approx 3.49$ : (a) Projection onto the $(V, n)$ plane. (b1)-(b2) Example time series of the solutions shown in panel (a). (b1) The sender (blue) remains silent whereas the receiver (red) spikes. (b2) Both the sender (blue) and the receiver (red) remain silent.

come an intense topic of research, both theoretically and experimentally. Indeed, one can envisage that this apparent violation of causality could in principle be exploited for engineering practical uses such as intelligent communication channels, and possibly in biological systems like neurons. The complete theoretical understanding of this process is still underway and the onward contribution of the present study is to append the view that canards, particularly in excitable systems, play a fundamental role.

Our insight that canards are involved in anticipation emerged as a consequence of the theoretical study by Ciszak et. al. ${ }^{4}$ that related anticipation to excitability threshold as well as our previous results, which identified excitability thresholds as maximal canards. Moreover, by employing the theoretical and numerical methodologies developed in our recent studies on canard-mediated synchronisation $^{24}$, we close the loop and demonstrate the role of canards and slow manifolds in mediating anticipation. Importantly, by suitable reduction of the unidirectional delayed coupling and under the weak-coupling assumption, we reveal that the coupling endows the receiver with an extra timescale separation thus inducing faster canard trajectories that predict the sender's trajectories.

We believe that the present study will contribute to the current discussion on correlation and causation ${ }^{54}$ and 


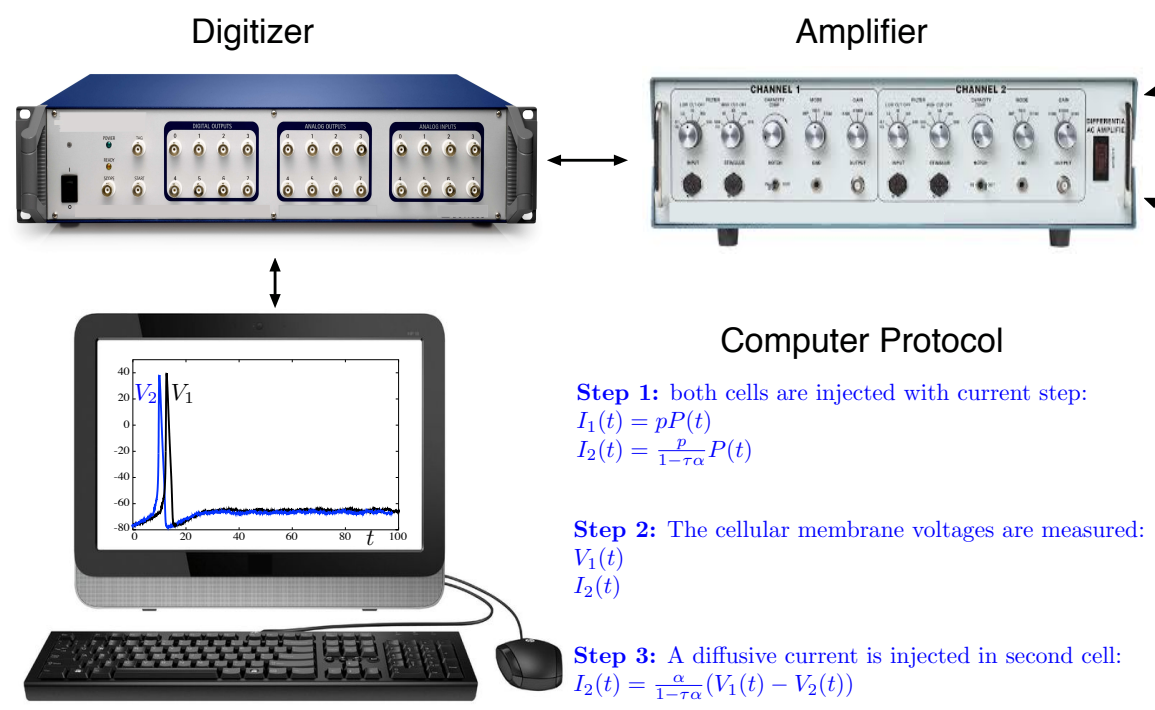

FIG. 12. Dynamic-clamp experimental protocole proposed to observe anticipation in real neurons.

how to better characterise information flow from a sender to a receiver in complex systems, e.g in biology, network dynamic, infectious disease transmitted between agents, etc. We highlight the need to consider canards to better understand correlation, causality and information transfer, particularly in complex excitable systems such as the Brain. In particular it will be useful in experimental protocols such as dual whole-cell electrophysiological recording, EEG/LFP measurements or fMRI voxel measurements, where there is a need to infer information transfer between communicating excitable nodes.

\section{CONCLUSION}

In this work, we have illustrated how a canardexplosive behavior subject to parameter variations, such as the input pulse amplitude $p$, the time delay $\tau$, and the coupling strength $\alpha$, can cause anticipation in coupled excitable systems. Moreover, we have shown that these canards can mediate both anticipation and AS, that is, for transients and asymptotic dynamics, respectively. The importance of transient dynamics in Neuroscience is critical since active matter (neural tissues) require fast responses (in reaction to environmental cues) and longterm (asymptotic) dynamics (or attractors in general) would have dramatic consequences to the survival of the organism; for more elements on the importance of transients in Brain dynamics, see the works of Friston 55-57. This is a natural consequence of the known facts that canards are sensitive to parameter variations and perturbations, and maximal canards in excitable slow-fast systems appear as boundaries between different oscillatory regimes such as non-spiking to spiking in neuron models. In these systems, the response of the sender-receiver to the perturbations depends on the regime where the solutions are located. In particular, the receiver dynam- ics is delayed if the perturbation keeps it longer along a repelling slow manifold. In the case of neuron models this scenario occurs for non-spiking solutions subject to perturbations. In contrast, a perturbation on the spiking solutions reduces the length of the trajectory along a repelling manifold, which yields an anticipated behavior for the receiver. Under very strong perturbations, the receiver can spike whereas the sender remains silent in the case of the sub-threshold solutions. The key to these results is showing the effects of the slow manifolds on the adjoint solutions to distinguish different responses of the system to delayed coupling. Our results successfully extends the work of Ref. 22 that predicted the existence of anticipated synchronous regime in synaptically interacting $\mathrm{HH}$ neurons. Moreover, we go beyond by predicting anticipation in the canard regime.

It is worth underlining that our study of canardmediated anticipation is by no means exhaustive. In particular, we outline the following key points: (1) We considered a restricted class of systems, namely multitimescale excitable systems and, in the case of the neuron models, this corresponds to type-II models, where the maximal canard is the best approximation of the excitability threshold. (2) We assume weak-coupling between the sender and the receiver and small delay in the coupling term, which enables the application of iPRC and the reduction of a DDE to an ODE. (3) A further key assumption is that both sender and receiver are at their common equilibrium point at the time the pulse is applied. Yet, we showed that if these key point holds, dynamical behaviors revealed under the pulse perturbation can persist in oscillatory regimes. If these conditions are not fulfilled, more complex scenarios could occur, leading or not to anticipation, due to the spiralling dynamics in the vicinity of this excitable equilibrium point. Finally, note that we do not consider noise in our setup. Adding noise could change the results, however depending on the 
signal-to-noise ratio, one could still have anticipation. If enough signal is available to determine the slow-fast nature of the intrinsic dynamics, then our results would still apply, meaning that noise will not necessarily destroy anticipation. Yet, noise adds to the sensitivity that is already due to the timescale separation, in a way that is less predictable. Canards are more robust in higher dimensions ${ }^{58}$, suggesting that anticipation and AS would be more predictable according to our results in that case. Such questions will be addressed in future work.

Despite these assumptions and reductions, a large class of coupled excitable systems can still be understood under this framework. The present work can be extended to tackle complex dynamics, for example by inspiring from the works of Refs. 40 and 41, on period-doubling (PD) bifurcations and mixed-mode oscillations (MMOs), for large-enough delay in canard cycles. We also plan to study canard-mediated anticipation using the framework of piecewise-linear slow-fast systems, so as to keep only the essentials of the dynamics while simplifying the model; this could also allow for a quantitative characterisation of the phenomenon ${ }^{58}$. Since we have mainly considered a pulse perturbation from a resting state and weak perturbations, our setting does not predict what occurs away from these settings. However, we have observed that unidirectionally delayed coupled canard cycles (with or without pulse perturbation) can give rise to PD bifurcations, MMOs and asynchronous solutions under relatively weak coupling and small delay. Further investigations, possibly with higher-order Taylor terms, are needed to clarify these effects. Moreover, follow-up studies will concern neuronal models that have bursting dynamics, such as the Hindmarsh-Rose or Plant models, with canard-mediated spike-adding dynamics and where anticipated spike synchronization ${ }^{8}$ could be studied. It is worth highlighting that in Ref. 22, type- $I$ excitable systems were shown not to display AS, which is consistent with our results since type-I models do not display canard solutions. However, adding more slow variables to type-I neuron models ${ }^{59}$ can give rise to bursting oscillations and generate canard dynamics, hence we envisage that the results of the present work on anticipation would apply to that case. Finally, we also proposed novel experiments in dynamic-clamp electrophysiological setting to investigate the predictions of our framework in neuronal systems.

\section{ACKNOWLEDGMENTS}

The authors thank Antonio E. Teruel for useful discussions. EKE was supported by the ERC Advanced Grant NerVi no. 227747. SR was supported by Ikerbasque (The Basque Foundation for Science) and from the Basque Excellence Research Centers (BERC).

\footnotetext{
${ }^{1}$ H. U. Voss, "Anticipating chaotic sychronization," Phys. Rev. E 61, 5115 (2000).
}

${ }^{2}$ M. Ciszak, O. Calvo, C. Masoller, C. R. Mirasso, and R. Toral, "Anticipating the response of excitable systems driven by random forcinf," Phys. Rev. Lett. 90, 204102 (2003).

${ }^{3}$ F. Matias, P. Carrelli, C. R. Mirasso, and M. Copelli, "Anticipated synchronization in a biologically plausible model of neuronal motifs," Phys. Rev. E 84, 021922 (2011).

${ }^{4}$ M. Ciszak, F. Marino, R. Toral, and S. Balle, "Dynamical mechanism of anticipating synchronization in excitable systems," Phys. Rev. Lett. 93, 114102 (2004).

${ }^{5}$ M. Hashemi, A. Valizadeh, and Y. Azizi, "Effect of duration of synaptic activity on spike rate of a hodgkin-huxley neuron with delayed feedback," Phys. Rev. E 85, 021917 (2012).

${ }^{6}$ F. S. Matias, L. L. Gollo, P. V. Carelli, S. L. Bressler, M. Copelli, and C. R. Mirasso, "Modeling positive granger causality and negative phase lag between cortical areas," NeuroImage 99, 411-418 (2014).

${ }^{7}$ C. R. Mirasso, P. Carrelli, T. Pereira, F. Matias, and M. Copelli, "Anticipated and zero-lag synchronization in motifs of delaycoupled systems," Chaos 27, 114305 (2017).

${ }^{8}$ T. Pyragiene and K. Pyragas, "Anticipating spike synchronization in nonidentical chaotic neurons," Nonlinear Dyn. 74, 297306 (2013).

${ }^{9} \mathrm{C}$. Masoller, "Anticipation in the synchronization of chaotic semiconductor lasers with optical feedback," Phys. Rev. Lett. 86, 2782-2785 (2001).

${ }^{10}$ M. Ciszak, C. R. Mirasso, R. Toral, and O. Calvo, "Predictprevent control method for perturbed excitable systems," Phys. Rev. E 79, 046203 (2009).

${ }^{11} \mathrm{H}$. Weia and L. Li, "Estimating paramters witg anticipating chaotic synchronization," Chaos 20, 023112 (2010).

${ }^{12}$ C. Mayol, C. R. Mirasso, and R. Toral, "Anticipated synchronization and the predict-prevent control method in the FitzHugh-Nagumo model system," Phys. Rev. E 85, 056216 (2012).

${ }^{13}$ S. Sivaprakasam, E. Shahverdiev, P. Spencer, and K. A. Shore, "Experimental demonstration of anticipating synchronization in chaotic semiconductor lasers with optical feedback," Physical Review Letters 87, 154101 (2001).

${ }^{14}$ A. N. Pisarchik, R. Jaimes-Reátegui, and J. H. García-López, "Synchronization of coupled bistable chaotic systems: experimental study," Philosophical Transactions of the Royal Society of London A: Mathematical, Physical and Engineering Sciences 366, 459-473 (2008).

${ }^{15} \mathrm{~J}$. N. Blakely, M. W. Pruitt, and N. J. Corron, "Time shifts and correlations in synchronized chaos," Chaos: An Interdisciplinary Journal of Nonlinear Science 18, 013117 (2008).

${ }^{16}$ Y. Liu, Y. Takiguchi, P. Davis, T. Aida, S. Saito, and J. Liu, "Experimental observation of complete chaos synchronization in semiconductor lasers," Applied Physics Letters 80, 4306-4308 (2002).

${ }^{17} \mathrm{~S}$. Tang and J. Liu, "Experimental verification of anticipated and retarded synchronization in chaotic semiconductor lasers," Physical review letters 90, 194101 (2003).

${ }^{18}$ N. J. Corron, J. N. Blakely, and S. D. Pethel, "Lag and anticipating synchronization without time-delay coupling," Chaos $\mathbf{1 5}$, 023110 (2005).

${ }^{19}$ L. M. Pecora and T. L. Carrol, "Synchronization in chaotic systems," Phys. Rev. Lett. 64, 821 (1990).

${ }^{20}$ L. M. Pecora and T. L. Carrol, "Driving systems with chaotic signals," Phys. Rev. A 44, 2374-2383 (1991).

${ }^{21} \mathrm{~K}$. Pyragas and T. Pyragiené, "Coupling design for a long-term anticipating synchronization of chaos," Phys. Rev. E 78, 0462217 (2008).

${ }^{22}$ F. Matias, P. Carrelli, C. R. Mirasso, and M. Copelli, "Anticipated synchronization in neuronal circuits unveiled by a phaseresponse-curve analysis." Phys. Rev. E 95, 052410 (2017).

${ }^{23}$ M. Desroches, M. Krupa, and S. Rodrigues, "Inflection, canards and excitability threshold in neuronal models," J. Math. Biol. 67, 989-1017 (2013).

${ }^{24}$ E. Köksal Ersöz, M. Desroches, and M. Krupa, "Synchroniza- 
tion of weakly coupled canard oscillators," Phys. D. 349, 46-61 (2017).

${ }^{25}$ H. B. Keller, Lectures on Numerical Methods in Bifurcation Problems (Springer Verlag, 1986).

${ }^{26}$ E. Benoît, J.-L. Callot, F. Diener, and M. Diener, "Chasse au canard," Collect. Math. 32, 37-119 (1981).

${ }^{27} \mathrm{M}$. Brøns, "Bifurcations and instabilities in the Greitzer model for compressor system surge," Math. Eng. Ind. 2, 51-63 (1988).

${ }^{28}$ S. A. Prescott, Y. De Koninck, and T. J. Sejnowski, "Biophysical basis for three distinct dynamical mechanisms of action potential initiation," PLoS Comput. Biol. 4, e1000198 (2008).

${ }^{29}$ P. De Maesschalck and M. Desroches, "Numerical continuation techniques for planar slow-fast systems," SIAM J. Appl. Dyn. Syst. 12, 1159-1180 (2013).

${ }^{30} \mathrm{~B}$. Ermentrout and D. Terman, Mathematical Foundations of Neuroscience (Springer, 2010).

${ }^{31} \mathrm{M}$. Diener, "The canard unchained or how fast/slow dynamical systems bifurcate," Math. Intell. 6, 38-48 (1984).

${ }^{32}$ W. Eckhaus, "Relaxation oscillations including a standard chase on french ducks," Lecture Notes in Math. 985, 449-494 (1983).

${ }^{33}$ F. Dumortier and R. Roussarie, "Canard cycles and center manifolds," Mem. Amer. Math. Soc. 577 (1996).

${ }^{34}$ M. Krupa and P. Szmolyan, "Extending geometric singular perturbation theory to nonhyperbolic points - fold and canard points in two dimensions,," SIAM J. Math. Anal. 33, 286-314 (2001).

${ }^{35}$ J. Mitry, M. McCarthy, N. Kopell, and M. Wechselberger, "Exitable neurons, firing threshold manifolds and canards," J. Math. Neurosci. 3 (2013).

${ }^{36}$ M. Krupa and P. Szmolyan, "Relaxation oscillation and canard explosion," J. Differ. Equ. 174, 312-368 (2001).

${ }^{37} \mathrm{M}$. Itoh and R. Tomiyasu, "Canards and irregular oscillations in a nonlinear circuit," IEEE International Symposium on Circuits and Systems (Singapore, 11-14 June 1991), 850-853 (1991).

${ }^{38} \mathrm{P}$. Strizhak and M. Menzinger, "Slow passage through a supercritical Hopf bifurcation: Time-delayed response in the BelousovZhabotinsky reaction in a batch reactor," J. Chem. Phys. 105, 10905 (1996).

${ }^{39}$ C. Chicone, "Inertial and slow manifolds for delay equations with small delays," J. Differ. Equ. 190, 364-406 (2003).

${ }^{40}$ S. A. Campbell, E. Stone, and T. Erneux, "Delay induced canards in high speed machining," Dyn. Syst. 24, 373-392 (2009).

${ }^{41}$ M. Krupa and J. D. Touboul, "Canard explosion in delay differential equations," J. Dyn. Diff. Equat. 28, 471-491 (2016).

${ }^{42}$ Y. Hayashi, S. J. Nasuto, and H. Eberle, "Renormalized time scale for anticipating and lagging synchronization," Phys. Rev. E 93, 052229 (2016).

${ }^{43}$ E. J. Doedel, A. R. Champneys, F. Dercole, T. Fairgrieve, Y. Kuznetsov, B. E. Oldeman, R. Paffenroth, B. Sandstede, X. J. Wang, and C. Zhang, "AUTO-07P: Continuation and bifurca- tion software for ordinary differential equations 2007,"

(2007), available at http://cmvl.cs. concordia.ca/auto.

${ }^{44} \mathrm{~S}$. Baer and T. Erneux, "Singular hopf bifurcation to relaxation oscillations," SIAM J. Appl. Math. 46, 721-739 (1986).

${ }^{45} \mathrm{~S}$. Baer and T. Erneux, "Singular hopf bifurcation to relaxation oscillations ii," SIAM J. Appl. Math. 52, 1651-1664 (1992).

${ }^{46} \mathrm{~J}$. Moehlis, "Canards for a reduction of Hodgkin-Huxley equations," J. Math. Biol. 52, 141-153 (2006).

${ }^{47}$ G. Ermentrout, Simulating, analyzing, and animating dynamical systems: a guide to XPPAUT for researchers and students (SIAM, 2002).

${ }^{48}$ A. Sharp, M. O'Neil, L. Abbott, and E. Mander, "The dynamic clamp: artificial conductances in biological neurons," Trends Neurosci. 6, 389-394 (1993).

${ }^{49}$ A. A. Prinz and R. Cudmore, "Dynamic clamp," Scholarpedia 6, $1470(2011)$.

${ }^{50}$ T. Bozhi, T. Cohen-Karni, Q. Qing, X. Duan, P. Xie, and C. M. Lieber, "Three-dimensional, flexible nanoscale field-effect transistors as localized bioprobes," Science 329, 830-834 (2010).

${ }^{51}$ J. Sieber, A. Gonzalez-Buelga, S. A. Nield, D. J. Wagg, and B. Krauskopf, "Experimental continuation of periodic orbits through a fold," Phys. Rev. Lett. 100, 244101 (2008).

${ }^{52}$ T. Netoff, M. Banks, A. Dorval, C. Acker, J. Haas, N. Kopell, and J. White, "Synchronization in hybrid neuronal networks of the hippocampal formation," J Neurophysiol. 93, 1197-1208 (2005).

${ }^{53}$ S. Wang, L. Chandrasekaran, F. Fernandez, J. White, and C. Canavier, "Short conduction delays cause inhibition rather than excitation to favor synchrony in hybrid neuronal networks of the entorhinal cortex," PLoS Comput. Biol. 8, 1002306 (2012).

${ }^{54}$ A. Messé, M.-T. Hütt, and C. C. Hilgetag, "Toward a theory of coactivation patterns in excitable neural networks," PLoS Comput. Biol. 14, e1006084 (2018).

${ }^{55}$ K. J. Friston, "The labile brain. i. neuronal transients and nonlinear coupling," Phil. Trans. R. Soc. B 355, 215-236 (2000).

${ }^{56}$ K. J. Friston, "The labile brain. ii. transients, complexity and selection," Phil.Trans. R. Soc. Lond. B 355, 237-252 (2000).

${ }^{57}$ K. J. Friston, "The labile brain. iii. transients and spatiotemporal receptive fields," Phil.Trans. R. Soc. Lond. B , 253-265 (2000).

${ }^{58}$ M. Desroches, A. Guillamon, E. Ponce, R. Prohens, S. Rodrigues, and A. E. Teruel, "Canards, folded nodes and mixed-mode oscillations in piecewise-linear slow-fast system," SIAM Rev. 58, 653-691 (2016).

${ }^{59}$ M. Desroches, M. Krupa, and S. Rodrigues, "Spike-adding in parabolic bursting: the role of folded-saddle canards," Phys. D 331, 58-70 (2016). 